\title{
Multifluid Block-Adaptive-Tree Solar wind Roe-type Upwind Scheme: Magnetospheric composition and dynamics during geomagnetic storms-Initial results
}

\begin{abstract}
A. Glocer, ${ }^{1}$ G. Tóth, ${ }^{2}$ Y. Ma, ${ }^{3}$ T. Gombosi, ${ }^{2}$ J.-C. Zhang, ${ }^{4}$ and L. M. Kistler ${ }^{4}$
Received 30 April 2009; revised 16 July 2009; accepted 19 August 2009; published 3 December 2009.

[1] The magnetosphere contains a significant amount of ionospheric $\mathrm{O}^{+}$, particularly during geomagnetically active times. The presence of ionospheric plasma in the magnetosphere has a notable impact on magnetospheric composition and processes. We present a new multifluid MHD version of the Block-Adaptive-Tree Solar wind Roe-type Upwind Scheme model of the magnetosphere to track the fate and consequences of ionospheric outflow. The multifluid MHD equations are presented as are the novel techniques for overcoming the formidable challenges associated with solving them. Our new model is then applied to the May 4, 1998 and March 31, 2001 geomagnetic storms. The results are juxtaposed with traditional single-fluid MHD and multispecies MHD simulations from a previous study, thereby allowing us to assess the benefits of using a more complex model with additional physics. We find that our multifluid MHD model (with outflow) gives comparable results to the multispecies MHD model (with outflow), including a more strongly negative Dst, reduced CPCP, and a drastically improved magnetic field at geosynchronous orbit, as compared to single-fluid MHD with no outflow. Significant differences in composition and magnetic field are found between the multispecies and multifluid approach further away from the Earth. We further demonstrate the ability to explore pressure and bulk velocity differences between $\mathrm{H}^{+}$and $\mathrm{O}^{+}$, which is not possible when utilizing the other techniques considered.
\end{abstract}

Citation: Glocer, A., G. Tóth, Y. Ma, T. Gombosi, J.-C. Zhang, and L. M. Kistler (2009), Multifluid Block-Adaptive-Tree Solar wind Roe-type Upwind Scheme: Magnetospheric composition and dynamics during geomagnetic storms-Initial results, J. Geophys. Res., 114, A12203, doi:10.1029/2009JA014418.

\section{Introduction}

[2] Since the earliest measurements of $\mathrm{O}^{+}$in the magnetosphere by Shelley et al. [1972], there has been a plethora of studies demonstrating its importance to understanding magnetospheric processes. These studies range from examinations of data from various satellites looking at composition and dynamics, to attempts to model the fate of $\mathrm{O}^{+}$in the magnetosphere. Understanding the contribution of different ion species and sources in magnetosphere is essential to developing our comprehension of the space environment.

[3] Studies based on the data from many separate satellites have established that ionospheric $\mathrm{O}^{+}$is a significant fraction of the magnetospheric plasma population, particularly during geomagnetic storms. Lennartsson et al. [1981] used the International Sun-Earth Explorer (ISEE) 1 satellite

\footnotetext{
${ }^{1}$ NASA GSFC, Greenbelt, Maryland, USA.

${ }^{2}$ Department of Atmospheric Oceanic and Space Science, University of Michigan, Ann Arbor, Michigan, USA.

${ }^{3}$ Institute of Geophysics and Planetary Physics, UCLA, Los Angeles, California, USA.

${ }^{4}$ Space Science Center, University of New Hampshire, Durham, New Hampshire, USA.
}

Copyright 2009 by the American Geophysical Union. 0148-0227/09/2009JA014418 to examine the composition of the magnetosphere within 15 Earth radii $\left(\mathrm{R}_{e}\right)$ during 10 geomagnetic storms. They found that $\mathrm{O}^{+}$can comprise $40 \%$, and as much as $80 \%$, of the total ion population, an unmistakable sign of the importance of the ionosphere as a plasma source to the magnetosphere. Using the Energetic Particle and Ion Composition (EPIC) instrument on the Geotail spacecraft, Nosé et al. [2003] examined the $\mathrm{O}^{+}: \mathrm{H}^{+}$energy density ratio in the plasma sheet during geomagnetic storms. They found that the ratio can reach $0.3-1.0$ at the peak of a storm; during the October 29-30, 2003 superstorm, the $\mathrm{O}^{+}: \mathrm{H}^{+}$energy density ratio reached 10-20 [Nosé et al., 2005]. Kistler et al. [2005] used Cluster data to examine the relative contribution of oxygen and hydrogen ions. They found that during a substorm the $\mathrm{O}^{+}: \mathrm{H}^{+}$ratio can reach as high as $10: 1$. There is even $\mathrm{O}^{+}$ present in the distant tail, albeit in small proportions, as GEOTAIL observations at $159 \mathrm{R}_{e}$ presented by Seki et al. [1996] demonstrate.

[4] Such a preponderance of data studies showing the strong contribution of $\mathrm{O}^{+}$points to the importance of the ionosphere in supplying the magnetosphere. In point of fact, by examining ISEE 1 satellite and Spacecraft Charging AT High Altitude (SCATHA) satellite mass spectrometer data, Sharp et al. [1985] found the ionosphere to be an important or dominant source of plasma for the inner magnetosphere. 
Additionally, Huddleston et al. [2005] found the ionosphere to be a sufficient source for magnetospheric plasma when studying data from Dynamics Explorer and Polar satellites.

[5] Such a large presence of ionospheric plasma has implications for magnetospheric processes such as the ring current. Shelley et al. [1972], for example, hypothesized that ionospheric outflow is important to storm time ring current. Daglis et al. [1999] compiled ring current composition from a number of satellite missions including the Active Magnetospheric Particle Tracer Explorer (AMPTE) mission and the Combined Release and Radiation Effects Satellite (CRRES) and showed that oxygen ions were more prevalent in the ring current during active periods.

[6] Modeling the effect that ionospheric outflow has on the magnetosphere is a challenging endeavor. Moore et al. [2007] approached this problem by using a particle tracing code to track millions of particles of various origin (solar wind, polar wind, auroral wind) through a magnetic field calculated by the Lyon-Fedder-Mobarry (LFM) [Lyon et al., 2004] ideal MHD model of the magnetosphere. They find that $\mathrm{O}^{+}$contributes a significant fraction of the inner magnetospheric pressure. Another approach, taken by Winglee et al. [2002], is to use a multifluid MHD model of the magnetosphere to track the fate of ionospheric plasma in the magnetosphere. Among their results is that the inclusion of ionospheric $\mathrm{O}^{+}$reduces the calculated cross polar cap potential. Glocer et al. [2009] couple a polar wind outflow model to a multispecies version of the BlockAdaptive-Tree Solar wind Roe-type Upwind Scheme (BATS-R-US) [Powell et al., 1999; De Zeeuw et al., 2000; Gombosi et al., 2001] magnetosphere model. Multispecies MHD solves the same equations as ideal MHD except that each ion species has its own continuity equation. This is in contrast to the multifluid approach in which each ion has its own continuity, momentum, and energy equation. Like the multifluid MHD technique, multispecies MHD allows for the ability to track the fate of ionospheric outflow, but the consequence of using this simpler technique is that physics associated with separate ion motion and pressure is not resolved. Using this approach for two geomagnetic storms, Glocer et al. [2009] found that ion outflow leads to a strengthening of the ring current, a reduction in the Cross Polar Cap Potential (CPCP), and a drastic improvement in the magnetic field at geosynchronous orbit (as compared to GOES data).

[7] The work presented in this paper builds on the study of Glocer et al. [2009] by replacing the multispecies MHD magnetosphere model with a newly developed multifluid MHD version of BATS-R-US. This lets us examine the ramifications of the additional physics included in the new approach, and allows us to attempt new studies not possible with the old approach. The multifluid MHD equations are described in section 2, as are new techniques for dealing with the unique challenges associated with solving them. Sections 3 and 4 present the results and analysis of our simulations.

\section{Model Details}

[8] In this study, the global magnetosphere is represented by the Block-Adaptive-Tree Solar wind Roe-type Upwind Scheme code. Other than ideal MHD and the multifluid
MHD developed in this study, BATS-R-US can solve the semirelativistic, Hall, and multispecies MHD equations. Explicit, implicit, and point-implicit time stepping are all available. Several total variation diminishing schemes are implemented with a finite volume discretization on a blockadaptive grids of both Cartesian and non-Cartesian geometries [Ma et al., 2007; Stout et al., 1997; Gombosi et al., 2001; Tóth et al., 2006, 2008].

[9] In this study we use a Cartesian grid. The computational domain of the magnetosphere model extends from $32 \mathrm{R}_{e}$ upstream to $224 \mathrm{R}_{e}$ downstream of the planet, and $64 \mathrm{R}_{e}$ to the sides. The inner boundary is a sphere of radius $2.5 \mathrm{R}_{e}$ centered on the Earth. The grid resolution varies from $1 / 4 \mathrm{R}_{e}$ near the inner boundary to $8 \mathrm{R}_{e}$ near the outer edges of the grid. This is a fairly coarse grid that allows fast computation on a relatively small number of CPUs. We set the solar wind conditions upstream of the planet using measurements by the ACE satellite.

[10] The BATS-R-US global magnetosphere model interacts with models representing other physical domains through the Space Weather Modeling Framework (SWMF) (see Toth et al. [2005] for details). The BATS-R-US inner boundary interacts with an ionosphere model represented by a 2D height integrated potential solver [Ridley et al., 2004], and with the Polar Wind Outflow (PWOM) model [Glocer et al., 2009]. The magnetosphere component can also interact with the inner magnetosphere component represented by the Rice Convection Model (RCM) to realistically capture the ring current pressure [De Zeeuw et al., 2004]. This last point is essential to modeling storm time conditions. These couplings are included in simulations presented in section 3 .

\subsection{Multifluid MHD Equations}

[11] Here we give a brief derivation for the multifluid MHD equations. The electron number density $n_{e}$ can be calculated from charge neutrality as

$$
n_{e}=\frac{1}{e} \sum_{s} n_{s} q_{s},
$$

where $e$ is the electron charge, $s$ indexes the ion fluids, $n_{s}$ is ion number density, and $q_{s}$ is the charge of ion fluid $s$.

[12] The momentum equations for the ion and electron fluids are

$$
\begin{gathered}
\frac{\partial \rho_{s} \mathbf{u}_{s}}{\partial t}+\nabla \cdot\left(\rho_{s} \mathbf{u}_{s} \mathbf{u}_{s}+I p_{s}\right)=n_{s} q_{s}\left(\mathbf{E}+\mathbf{u}_{s} \times \mathbf{B}\right)+S_{\rho_{s} \mathbf{u}_{s}} \\
\frac{\partial \rho_{e} \mathbf{u}_{e}}{\partial t}+\nabla \cdot\left(\rho_{e} \mathbf{u}_{e} \mathbf{u}_{e}+I p_{e}\right)=-n_{e} e\left(\mathbf{E}+\mathbf{u}_{e} \times \mathbf{B}\right)+S_{\rho_{e} \mathbf{u}_{e}}
\end{gathered}
$$

where $\rho_{s}$ and $\rho_{e}$ are the ion and electron mass densities, $\mathbf{u}_{s}$ and $\mathbf{u}_{e}$ are the ion and electron velocities, $p_{s}$ and $p_{e}$ are the ion and electron pressures, $\mathbf{E}$ and $\mathbf{B}$ are the electric and magnetic field strengths, finally $S_{\rho_{s} \mathbf{u}_{s}}$ and $S_{\rho_{e} \mathbf{u}_{e}}$ are arbitrary source terms due to electron-ion collisions, charge exchange for ions, etc.

[13] We can use the electron momentum equation (3) to express the electric field. Since the electron mass is much smaller than the ion mass, we may neglect terms propor- 
tional to the electron mass density and arrive at the generalized Ohm's law

$$
\mathbf{E}=-\mathbf{u}_{e} \times \mathbf{B}-\frac{1}{e n_{e}} \nabla p_{e}+\eta \mathbf{J},
$$

where $\eta \mathbf{J}$ is the resistivity term originating from the source term $S_{\rho_{e}} \mathbf{u}_{e}$ in the electron momentum equation. The current density can be written as

$$
\mathbf{J}=-e n_{e} u_{e}+\sum_{s} q_{s} n_{s} \mathbf{u}_{s} .
$$

This can be rearranged to obtain the electron velocity as

$$
\mathbf{u}_{e}=-\frac{\mathbf{J}}{e n_{e}}+\mathbf{u}_{+},
$$

where

$$
\mathbf{u}_{+}=\frac{\sum_{s} n_{s} q_{s} \mathbf{u}_{s}}{e n_{e}}
$$

is the charge averaged ion velocity. We can substitute the electron velocity equation (6) into Ohm's law (4) to obtain the electric field, which is then substituted into the ion momentum equation (2) and the induction equation to arrive at the following multifluid MHD equations:

$$
\begin{gathered}
\frac{\partial \rho_{s}}{\partial t}+\nabla \cdot\left(\rho_{s} \mathbf{u}_{s}\right)=S_{\rho_{s}}, \\
\frac{\partial \rho_{s} \mathbf{u}_{s}}{\partial t}+\nabla \cdot\left(\rho_{s} \mathbf{u}_{s} \mathbf{u}_{s}+I p_{s}\right)=n_{s} q_{s}\left(\mathbf{u}_{s}-\mathbf{u}_{+}\right) \times \mathbf{B} \\
+\frac{n_{s} q_{s}}{n_{e} e}\left(\mathbf{J} \times \mathbf{B}-\nabla p_{e}\right)+S_{\rho_{s} \mathbf{u}_{s}}, \\
\frac{\partial p_{s}}{\partial t}+\nabla \cdot\left(p_{s} \mathbf{u}_{s}\right)=-(\gamma-1) p_{s} \nabla \cdot \mathbf{u}_{s}+S_{p_{s}}, \\
\frac{\partial \mathbf{B}}{\partial t}-\nabla \times\left(\mathbf{u}_{+} \times \mathbf{B}\right)=0,
\end{gathered}
$$

where $\gamma$ is the adiabatic index ( $=5 / 3$ in this study). The units of the magnetic field are chosen such that magnetic permeability of vacuum $\mu_{0}$ is unity. We note the following: (1) the multi-ion MHD equations (8)-(11) cannot be written into conservation form, (2) resistivity is neglected, and (3) the Hall term and the gradient of the electron pressure are neglected in the induction equation (11) to avoid the stiffness due to Whistler waves. BATS-R-US can include resistivity as well as the Hall term [Tóth et al., 2008], but we will not use these features in this study.

[14] The electron pressure can either be assumed to be some fraction of the total ion pressure $\left(p_{e}=\alpha \sum_{s} p_{s}\right)$ or we can solve the electron pressure equation

$$
\frac{\partial p_{e}}{\partial t}+\nabla \cdot\left(p_{e} \mathbf{u}_{e}\right)=-(\gamma-1) p_{e} \nabla \cdot \mathbf{u}_{e}+S_{p_{e}}
$$

where the source term may include the electron heat conduction. In this study we use an electron pressure equal to $20 \%$ of the ion pressure. This number was chosen to be consistent with inner magnetosphere simulations (M.-C. Fok, private correspondence, 2009), and the results were found to be insensitive to a variation of $\pm 10 \%$.

[15] We have also implemented the option of solving for the hydrodynamic energy density $e_{s}=\rho_{s} \mathbf{u}_{s}^{2} / 2+p_{s} /(\gamma-1)$ as

$$
\begin{aligned}
& \frac{\partial e_{s}}{\partial t}+\nabla \cdot\left[\left(e_{s}+p_{s}\right) \mathbf{u}_{s}\right]=\mathbf{u}_{s} \\
& \cdot\left[n_{s} q_{s}\left(\mathbf{u}_{s}-\mathbf{u}_{+}\right) \times \mathbf{B}+\frac{n_{s} q_{s}}{n_{e} e}\left(\mathbf{J} \times \mathbf{B}-\nabla p_{e}\right)\right]+S_{e_{s}}
\end{aligned}
$$

instead of using equation (10). While equation (13) is not fully conservative, it can give roughly correct jump conditions across shocks when the magnetic energy density is small relative to the kinetic and thermal energy densities.

[16] The source terms in equations (8)-(13), denoted by $S_{\rho_{s}}, S_{\rho_{s} \mathbf{u}_{s}}, S_{p_{s}}, S_{p_{e}}$, and $S_{e_{s}}$ represent the mass, momentum, pressure or energy sources, respectively. They may contain charge exchange, recombination, photoionization, chemistry, ion-ion collisions, etc. These source terms are negligible when simulating the Earth's magnetosphere, but they are quite substantial when simulating other problem types, such as comets or ionospheres of unmagnetized planets and moons.

[17] It is a usual practice in magnetospheric MHD codes to use a semirelativistic correction with the actual or an artificially reduced speed of light [see Gombosi et al., 2001, and references therein]. This so-called Boris correction [Boris, 1970] limits the Alfvén speed to the speed of light as demanded by the theory of relativity. The artificial light speed reduction is done to further reduce the Alfvén speed so that the equations are less stiff. This idea can be extended to the multifluid MHD equations too, although we are not attempting a rigorous derivation here. Since the multifluid MHD equations cannot be written in conservative form with respect to magnetic field, one can use the simplest form of the Boris modification by changing the number densities on the right hand sides of the momentum and energy equations (9) and (13) as

$$
n_{s}^{\prime}=\frac{n_{s}}{1+v_{A}^{2} / c^{\prime 2}},
$$

where $v_{A}^{2}=\mathbf{B}^{2} / \rho$ is the Alfvén speed squared and $c^{\prime}$ is the actual or modified (lowered) speed of light. Although we have not attempted a formal derivation, it seems reasonable to use the same factor for all ion fluids as in the Boris correction of the single ion MHD equations. Based on our numerical simulations, the correction works as expected: it allows larger explicit time step and it reduces the numerical diffusion. The simulations presented in this paper use the Boris correction with the speed of light reduced by a factor of 0.02 .

[18] Two-stream instabilities physically restrict the relative velocities of the ions parallel to the magnetic field line. This subgrid process is difficult to directly include into our model. We therefore mimic its effect by including a nonlinear artificial friction term to limit the relative 
velocities to realistic values, rather than directly simulate the instability. In particular we used the following source term in the momentum equation:

$$
S_{\rho \mathbf{u}_{s}}^{\text {friction }}=\frac{1}{\tau_{c}} \sum_{q \neq s} \min \left(\rho_{s}, \rho_{q}\right)\left(\mathbf{u}_{q}-\mathbf{u}_{s}\right)\left(\frac{\left|\mathbf{u}_{s}-\mathbf{u}_{q}\right|}{u_{c}}\right)^{\alpha_{c}},
$$

where $q$ indexes all the other fluids, $\tau_{c}$ is the cutoff time scale, $u_{c}$ is the cutoff velocity and $\alpha_{c}$ is the cutoff exponent. The larger the exponent, the sharper the cutoff is for velocity differences nearing or exceeding $u_{c}$. We take the minimum of the two densities instead of their product so that the friction term is equally effective in regions of low and high densities.

[19] While this is a rather ad hoc formula, it achieves some control over the velocity differences. Without the friction term the velocity differences grow to unrealistically high values. Currently we set $\tau_{c}, u_{c}$ and $\alpha_{c}$ as input parameters for the run, and hold them constant. In the future we will explore more physics based parameter settings and friction formulas. The simulations in section 3 set $u_{c}=50 \mathrm{~km} / \mathrm{s}, \tau_{c}=10$ minutes, and $\alpha_{c}=4$.

\subsection{Algorithm and Techniques}

[20] Solving the multifluid MHD equations presented in the previous section, is not without significant challenges. This necessitates the development of new algorithms and techniques. In this subsection we describe how we deal with issues of stability and two-stream instability.

[21] The issue of stability arises when trying to treat the "stiff" multiion source term in the ion momentum equation. To ensure stability, while still being able to take reasonable time steps, we evaluate this term in a pointimplicit manner

$$
\begin{gathered}
\left(\rho_{s} \mathbf{u}_{s}\right)^{n+1}=\left(\rho_{s} \mathbf{u}_{s}\right)^{n}-\Delta t \nabla \cdot \mathbf{F}^{n} \\
+\Delta t\left[\frac{q_{s}}{M_{s}}\left(\rho_{s} \mathbf{u}_{s}-\rho_{s} \mathbf{u}_{+}\right)^{n+1} \times \mathbf{B}^{n}+\frac{n_{s}^{n} q_{s}}{n_{e}^{n} e}\left(\mathbf{J}^{n} \times \mathbf{B}^{n}-\nabla p_{e}^{n}\right)\right]
\end{gathered}
$$

$$
+\Delta t S_{\rho \mathbf{u}_{s}}^{\text {friction, } n+1}+\Delta t S_{\rho \mathbf{u}_{s}}^{\text {other }}
$$

where $M_{s}$ is the atomic/molecular mass of species $s$. Notice that the term $\rho_{s} \mathbf{u}_{s}-\rho_{s} \mathbf{u}_{+}$and the friction source term $S_{\rho \mathbf{u}_{s}}^{\text {friction }}$ are evaluated at time level $n+1$ instead of $n$. Only the momenta are treated implicitly, so we have to invert a $3 N$ by $3 N$ matrix, where $N$ is the number of fluids. The matrix elements are calculated analytically for sake of efficiency and accuracy. If there was no friction term, one could also invert 3 separate $N \times N$ matrices, since the three spatial components of the $\rho_{s} \mathbf{u}_{s}-\rho_{s} \mathbf{u}_{+}$are not coupled with each other. The friction term (15), however, couples the three components for $\alpha_{c} \neq 0$. If there are other stiff source terms, e.g. due to chemical reaction, we also include them into the point-implicit scheme.

[22] The previously described methods have all been implemented in BATS-R-US. The next section will describe applications of our new model to the Earth's magnetosphere system.

\section{Results}

[23] Ionospheric $\mathrm{O}^{+}$is a strong contributor to the storm time magnetosphere's composition [Fu et al., 2001]. Such periods are therefore ideal for exercising our multifluid approach that can track relative ion composition, velocity, pressure and associated effects. We test our multifluid MHD model of the magnetosphere by simulating two geomagnetic storms: May 4, 1998 and March 31, 2001. In this section we describe the model setup, present the results of our simulation, and compare them with single index measures (Dst and CPCP) and with specific satellites (GOES and Cluster). Comparisons to other approaches of modeling the magnetosphere are included whenever possible.

[24] The simulated events, May 4, 1998 and March 31, 2001, are very strong geomagnetic events. The minimum Dst reaches as low as $-200 \mathrm{nT}$ and $-400 \mathrm{nT}$, respectively. The solar wind conditions are given in Figures 1 and 2 . These solar wind conditions are used to set the upstream boundary condition for our simulations.

[25] We use the Space Weather Modeling Framework (SWMF) in order to simulate these events. By using the SWMF, rather than just the multifluid BATS-R-US, we take advantage of input from other parts of the space environment system. The SWMF is configured with the following 4 components: (1) Global Magnetosphere (GM), represented by the multifluid BATS-R-US model described in the previous section; (2) Ionosphere Electrodynamics (IE), represented by a vertical potential solver [Ridley et al., 2004; Wang et al., 2008]; (3) Inner Magnetosphere(IM), represented by the Rice Convection Model (RCM) [De Zeeuw et al., 2004]; and (4) Polar Wind (PW), represented by the Polar Wind Outflow Model (PWOM) [Glocer et al., 2009]. The GM component interacts with i.e.component at the inner boundary, passing field-aligned currents and receiving back the ionospheric potential. The PW component also obtains the ionospheric potential from i.e.and uses it to advect the field lines. The PW component calculates the densities, momenta and pressures of ionospheric $\mathrm{H}^{+}$and $\mathrm{O}^{+}$at its upper boundary and passes them to the GM component. GM uses these values to set the inner boundary conditions in the polar region. The details of the PW calculated outflow are left to the work of Glocer et al. [2009], allowing us to focus on the magnetosphere model developed in this paper. The IM model receives the ionospheric potential from IE, and field line volumes from the GM component. IM returns the pressure in the inner magnetosphere to GM. All these models run concurrently in the SWMF and information is exchanged between them every 10 s of simulation time. We ran our model on NASA Advanced Supercomputing's (NAS) Pleiades supercomputer. Using 64 processors, we can simulate 12 hours of a geomagnetic storm in about 13.7 hours: about $88 \%$ of real time.

[26] The storms we chose allow us to directly compare the multifluid MHD approach developed in this paper with the approaches presented by Glocer et al. [2009]. That study uses the same SWMF setup but there the GM component is represented by a multispecies MHD BATS-R-US model 


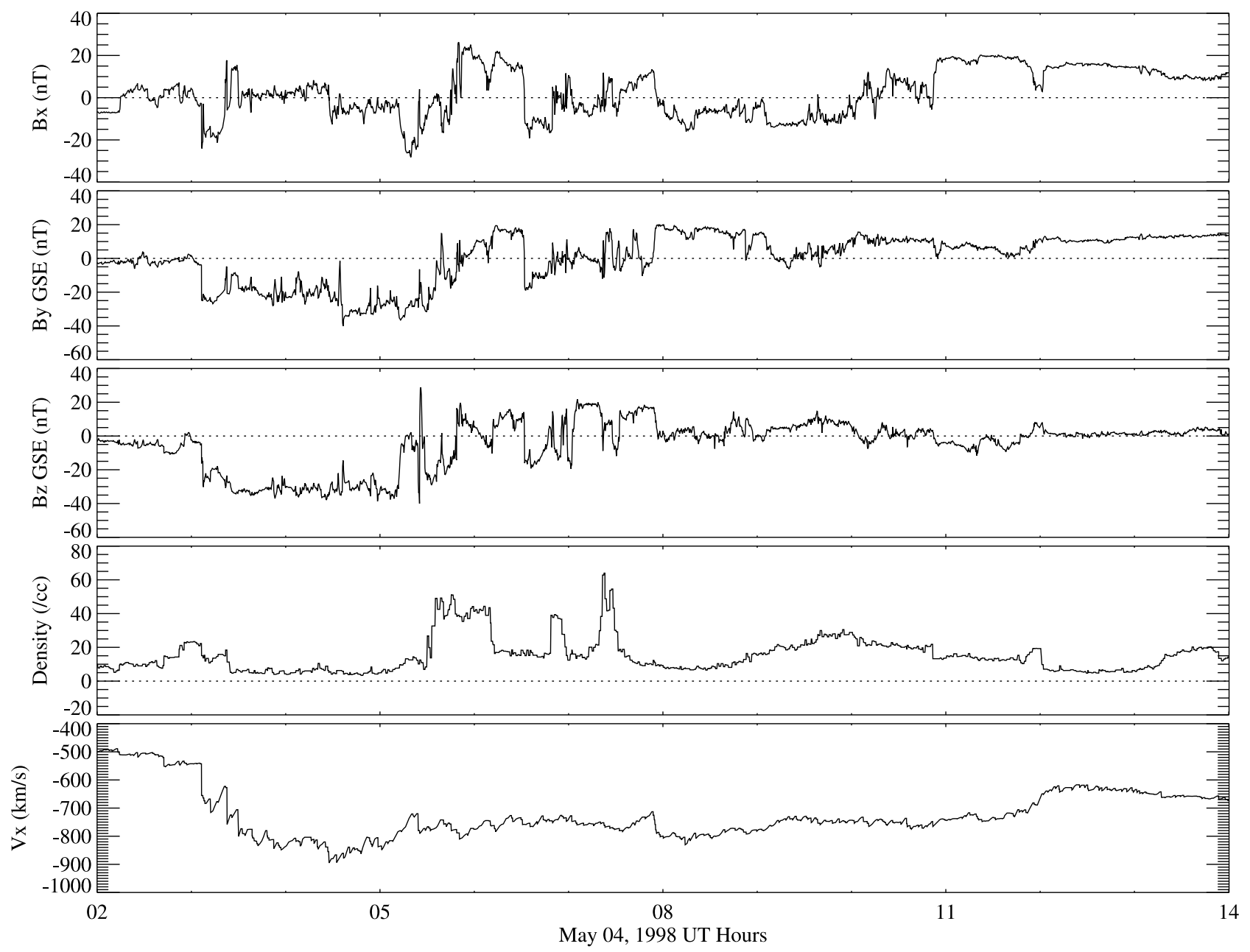

Figure 1. Solar wind condition used to set the upstream boundary condition for the magnetosphere for the May 4, 1998 storm.

which uses separate continuity equations for $\mathrm{O}^{+}$and $\mathrm{H}^{+}$but uses common momentum and energy equations. We also include the ideal MHD reference solution without ionospheric outflow as used by Glocer et al. [2009]. We compare the multifluid MHD technique with the multispecies MHD and single-fluid MHD whenever possible so as to illustrate the similarities and differences between the disparate approaches. Moreover, by comparing with approaches that include less physics, we can gain a better understanding of what is needed to model various processes. A careful reader may notice that the multispecies results used as a reference in this study differ somewhat from what is shown by Glocer et al. [2009]. The differences are due to a minor error in the prior study's calculation of FACs that has now been corrected (the corrected results are shown here).

[27] Having described the geomagnetic storms under consideration, the simulation setup, and the reference simulations to be compared with, we turn our attention to the simulation results, beginning with the Dst and Cross Polar Cap Potential indices. In our model, the simulated Dst is calculated by evaluating the Biot-Savart integral for the center of the Earth from all currents in the modeled domain. Figures 3 and 4 show the simulated Dst and CPCP indices, respectively. The comparison for the May 4, 1998 event and the comparison for the March 31, 2001 event are shown. Included in Figures 3 and 4 are the measured Dst data, and the AMIE (Assimilative Mapping of Ionosphere Electrodynamics) [Ridley and Kihn, 2004] CPCP values. The multifluid and multispecies results give quite comparable results for these global indices, including more strongly negative Dst values and lower CPCP values compared to the singlefluid MHD reference solution, for both events. The Dst obtained with ion outflow agrees better with observations than the Dst obtained without ion outflow.

[28] We now turn our attention from the large scale indices to specific satellite comparisons. In particular, we focus on how the simulated magnetic field at geosynchronous orbit compares to the field measured by specific GOES satellites. Figures 5 to 6 compares the simulated magnetic field to GOES magnetic field data for the May 4, 1998 and March 31, 2001 events. Figures 5a, 5b, 6a, and 6b are multifluid MHD results with ion outflow; Figures 5c, 5d, $6 \mathrm{c}$, and $6 \mathrm{~d}$ are the standard single-fluid MHD results with no ion outflow. Figures 5 and 6 show that the multifluid MHD simulations with ionospheric outflow compare much better with data than the reference solution. Table 1 summarizes the root-mean-square (RMS) error in the magnetic field magnitude and elevation angle for multifluid, multi- 


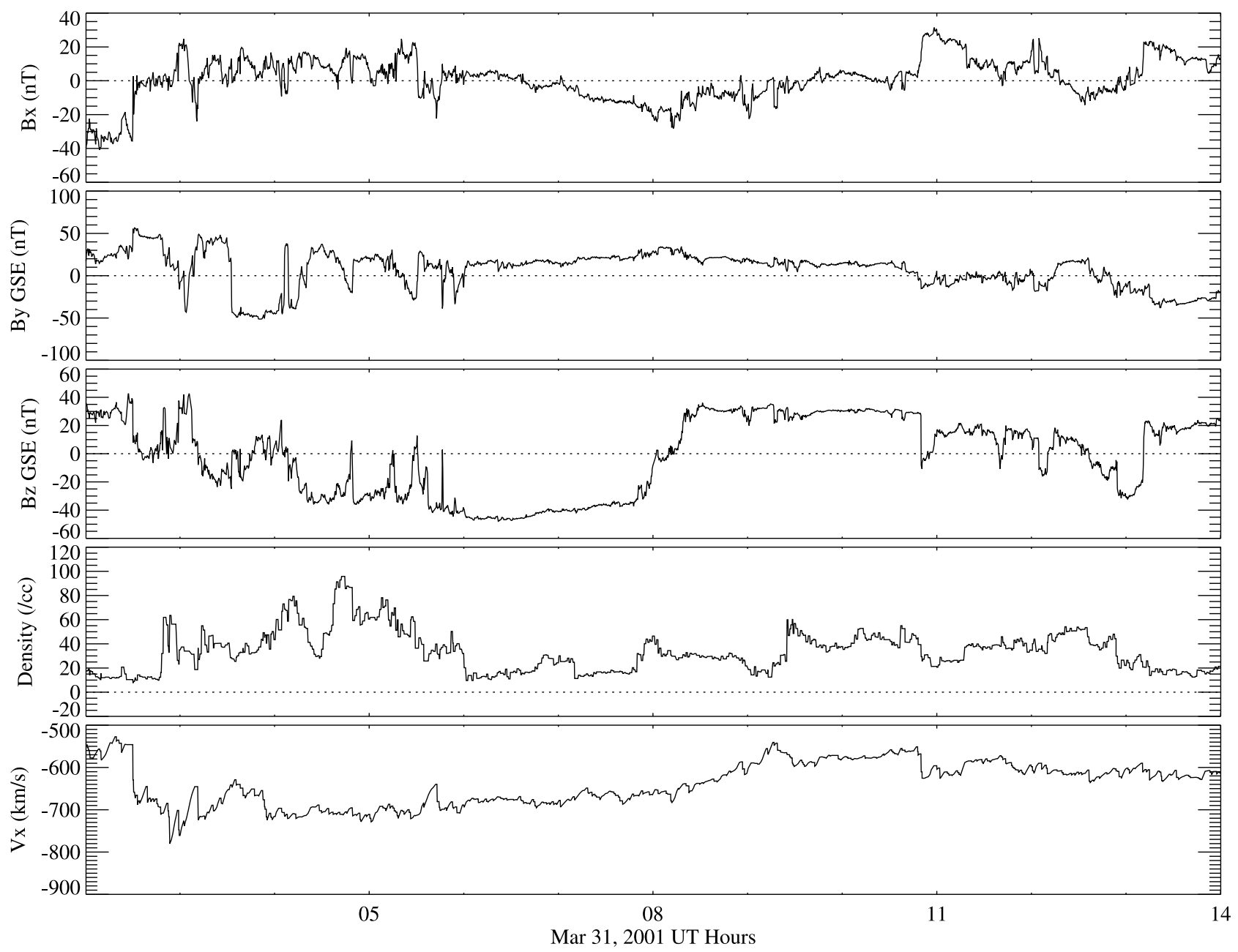

Figure 2. Solar wind condition used to set the upstream boundary condition for the magnetosphere for the March 31, 2001 storm.
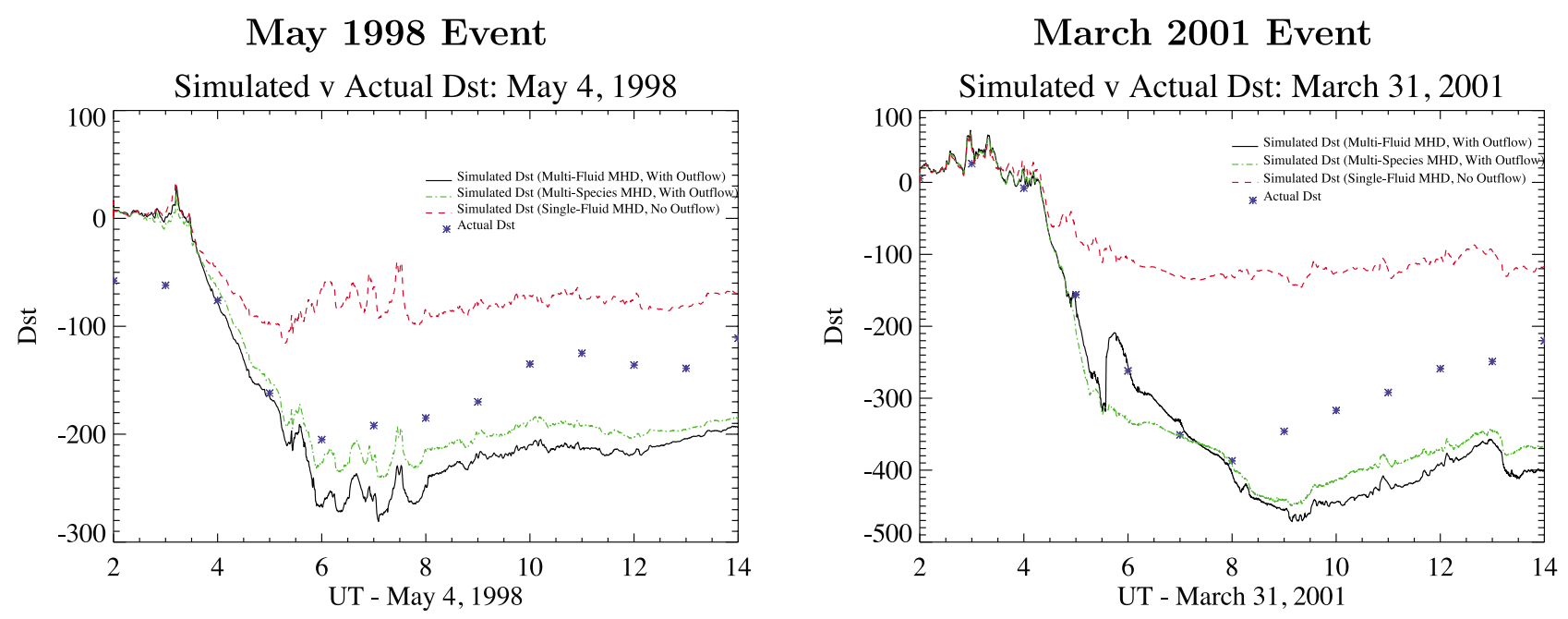

Figure 3. A comparison of the simulated Dst with Dst data. Multifluid MHD and multispecies MHD results (with ion outflow) are shown as are single-fluid MHD (without ion outflow) simulation results. 
May 1998 Event

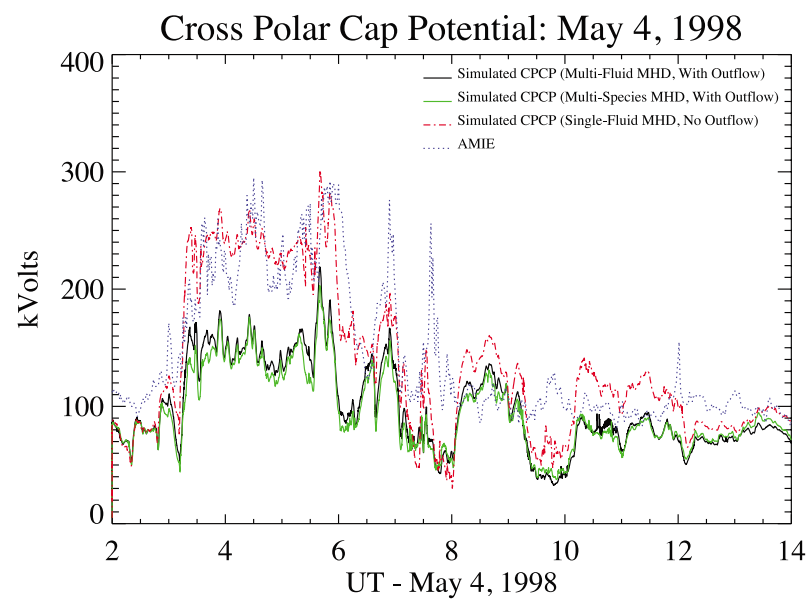

March 2001 Event

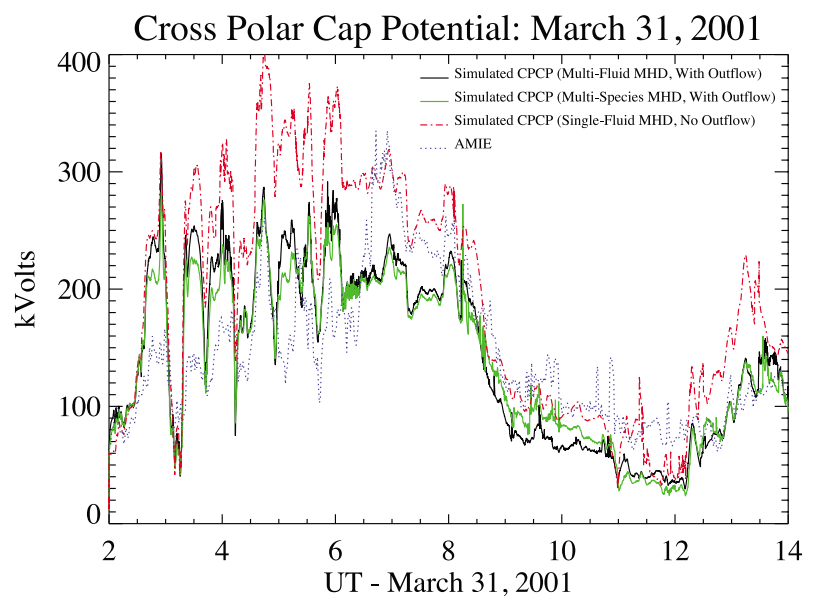

Figure 4. A comparison of the simulated northern CPCP with the AMIE [Ridley and Kihn, 2004] derived CPCP. Multifluid MHD and multispecies MHD results (with ion outflow) are shown as are single-fluid MHD (without ion outflow) simulation results.

species, and our reference MHD solution. Again, the multifluid and multispecies MHD results with ion outflow give significantly improved results, as compared to GOES measurements, than the reference MHD solution without outflow. The reason for the improvement, given by Glocer et $a l$. [2009], is the strengthening of the ring current due to ionospheric particles making their way into the inner magnetosphere.

[29] We verify the simulated composition results for the March 31, 2001 event by directly comparing with the ion composition measurements from the CIS/CODIF instrument on the Cluster 1 satellite. The CODIF instrument measures ion composition over the energy range $40 \mathrm{eV}$ to $40 \mathrm{keV}$ [Rème et al., 2001]. Cluster/CODIF data are not available for the May 4, 1998 event. Figure 7 shows $\mathrm{O}^{+}$and $\mathrm{H}^{+}$ density comparisons with Cluster/CODIF data for both multifluid MHD (left) and the multispecies MHD (right) from Glocer et al. [2009]. Both approaches find the peak in the $\mathrm{O}^{+}$density, although it is underestimated, and the simulations are generally in line with the data. The $\mathrm{H}^{+}$ density agrees relatively well until about 10:30 UT. At this time the spacecraft probably enters the lobe region. At 11:45 UT the Cluster satellite is definitely in the magnetosheath, while in the model the satellite is probably at the edge of the magnetosphere which is smeared out by the finite numerical resolution. The Cluster $\mathrm{H}^{+}$density in the magnetosheath is too low in this case because it has not been corrected for the dead time that occurs for high rates. The single-fluid MHD is not included in this comparison as it lacks composition information.

[30] Thus far, the multifluid and multispecies MHD approaches appear to give similar results, but they begin to diverge when examining composition and magnetic field topology on a larger scale. Figure 8 shows the percentage of $\mathrm{O}^{+}$in the magnetosphere along with magnetic field traces at times during the March 31, 2001 event. The $\mathrm{y}=0$ plane is shown. Multifluid MHD results are shown as are multispecies MHD simulations from Glocer et al. [2009] included for comparison. The four time periods shown correspond to a time near the beginning of the simulation (3 UT), a period of strongly negative Bz (7 UT), a time of strongly positive $\mathrm{Bz}$ (10 UT), and a time near the end of the simulation (13 UT). The multifluid and multispecies MHD results are similar to each other in terms of overall magnetic topology and composition near the planet, however significant differences are visible further from the planet. In particular, there is much less oxygen present further down the tail in the multifluid MHD simulations than in the multispecies MHD simulations. Moreover, the magnetic topology down the tail, and near the subsolar point, exhibit significant differences. Similar results were found for the May 4, 1998 event, but are not shown.

[31] Multifluid MHD allows us to address topics that are simply not accessible using multispecies MHD or single fluid MHD, such as the separate ion velocities and pressures. Figure 9 compares the $\mathrm{O}^{+}$and $\mathrm{H}^{+}$simulated velocity measurements to Cluster/CODIF data. The velocity comparison demonstrate significant room exists for improvement. In general the $\mathrm{H}^{+}$simulated velocities agree better with the Cluster/CODIF measurements than the $\mathrm{O}^{+}$simulated velocities. When restricting the simulated values to the sensitivity range of the instrument, the comparisons improve somewhat. The most visible problem with the velocities is strong tailward flow in the simulated velocities as Cluster passes into the near tail. It is unsurprising that the velocity moment of the Cluster/CODIF data provides the worst

Figure 5. Data model comparisons of magnetic field from (a and c) GOES 08 and (b and d) GOES 09 with the MHD magnetic field calculated by BATS-R-US for the May 4, 1998 event. The data are in blue and the simulations are in black. Figures $5 \mathrm{a}$ and $5 \mathrm{~b}$ are from a multifluid MHD simulation with outflow and Figures $5 \mathrm{c}$ and $5 \mathrm{~d}$ are from a single-fluid MHD simulation without outflow. The root mean square error (RMSE) is indicated along the right-hand side. 
(a) Multi-Fluid MHD
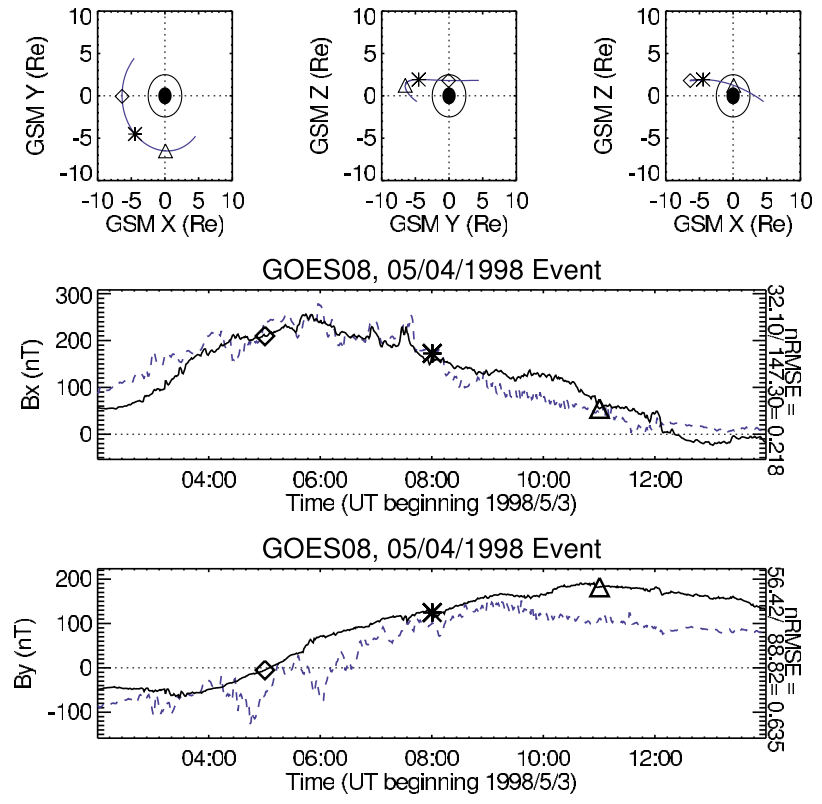

GOES08, 05/04/1998 Event
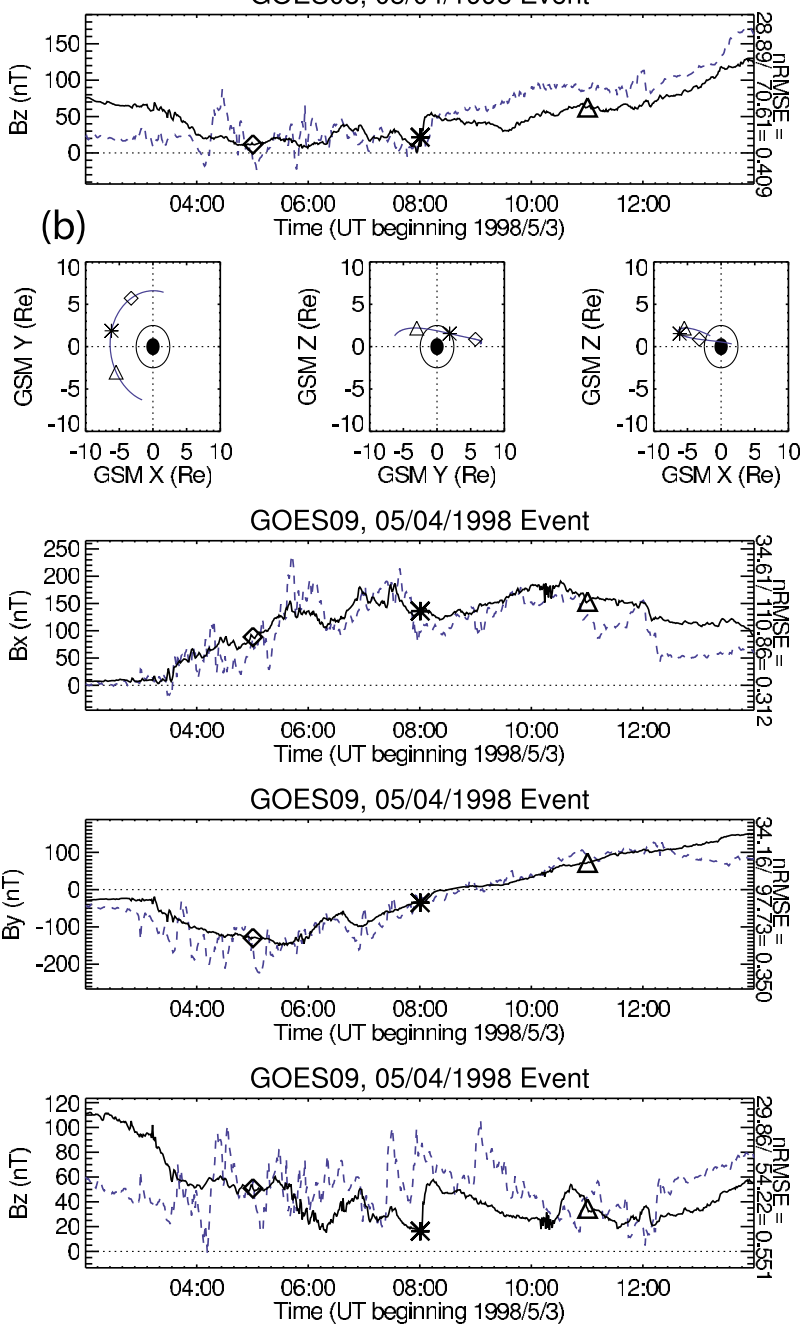

(c) Single-Fluid MHD
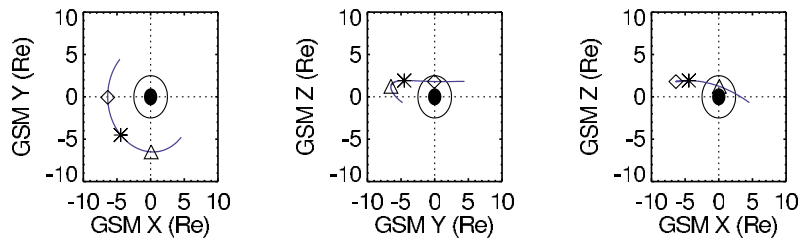

GOES08, 05/04/1998 Event

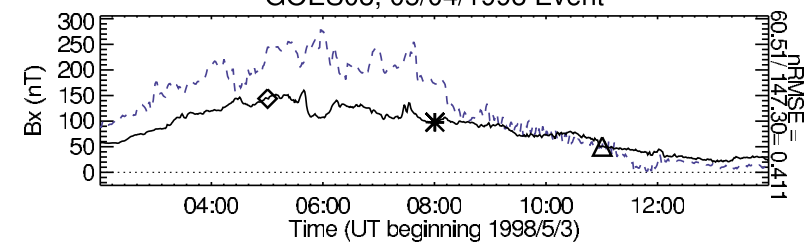

GOES08, 05/04/1998 Event

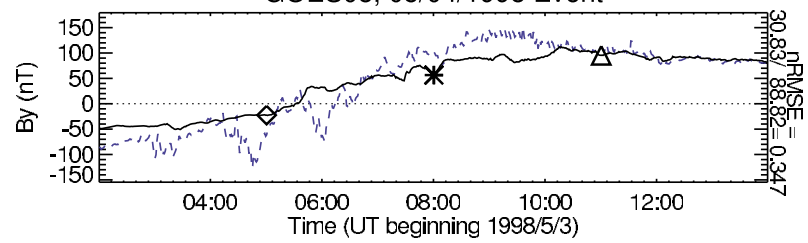

GOES08, 05/04/1998 Event
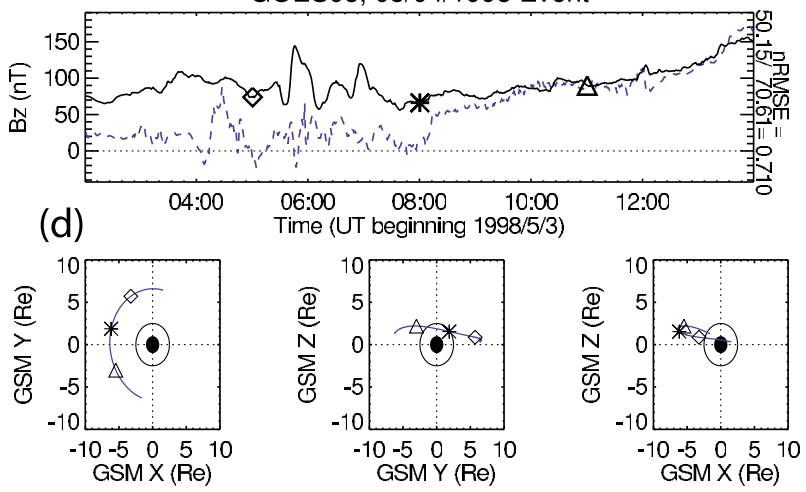

GOES09, 05/04/1998 Event

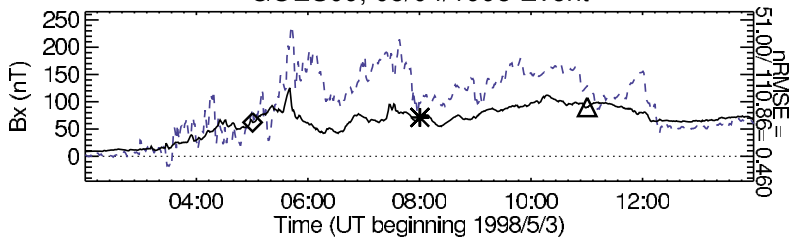

GOES09, 05/04/1998 Event
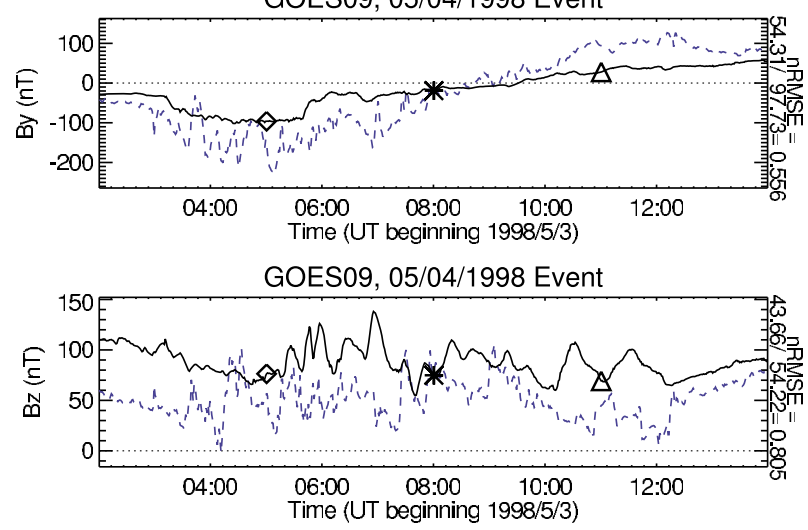

Figure 5 
(a) Multi-Fluid MHD
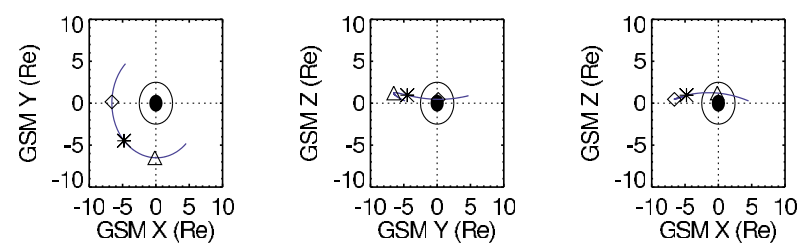

GOES08, 03/31/2001 Event

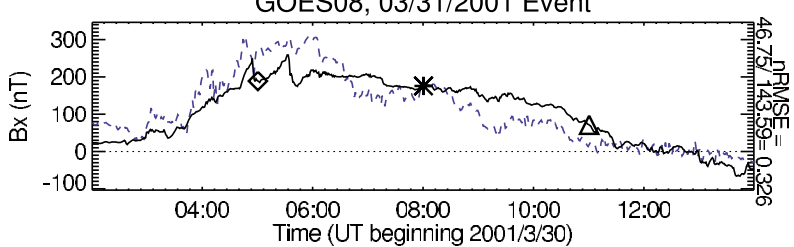

GOES08, 03/31/2001 Event

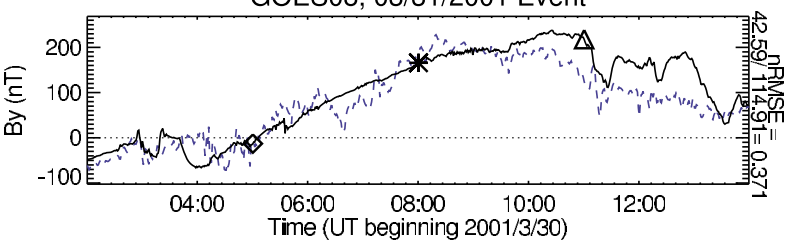

GOES08, 03/31/2001 Event
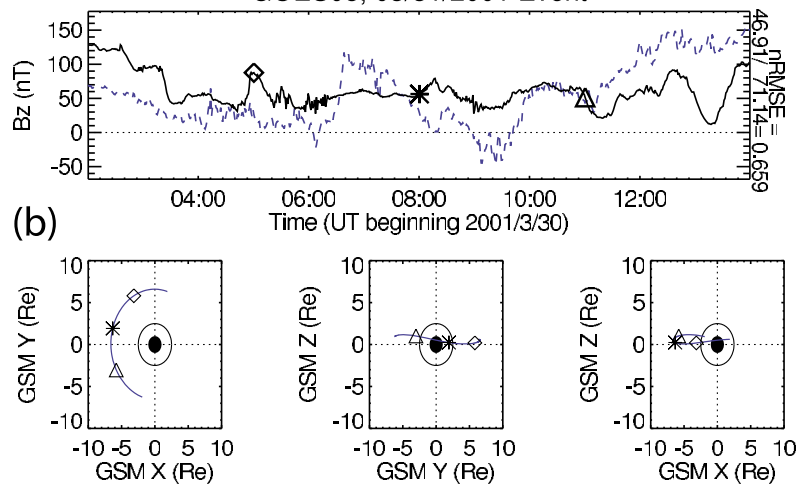

GOES10, 03/31/2001 Event

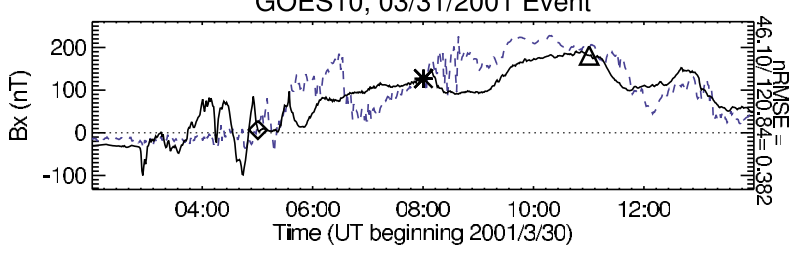

GOES10, 03/31/2001 Event

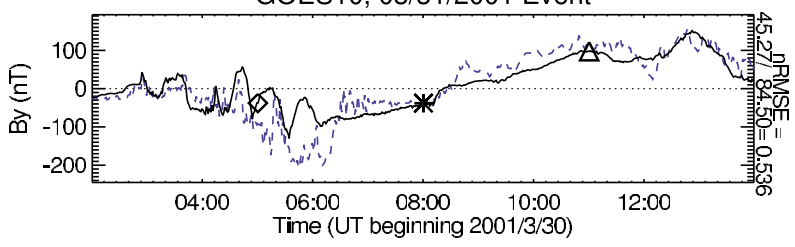

GOES10, 03/31/2001 Event

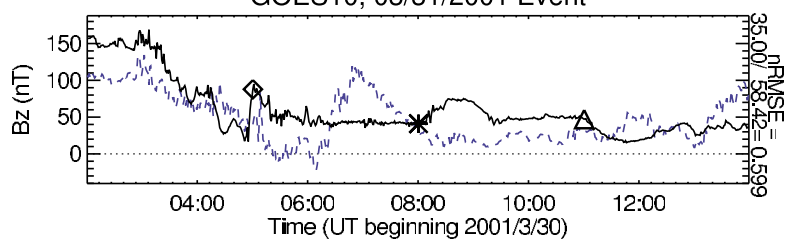

(c) Single-Fluid MHD
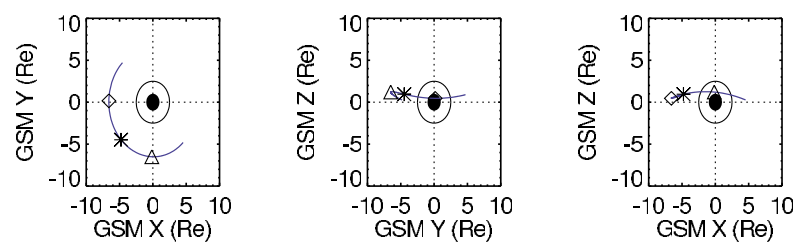

GOES08, 03/31/2001 Event

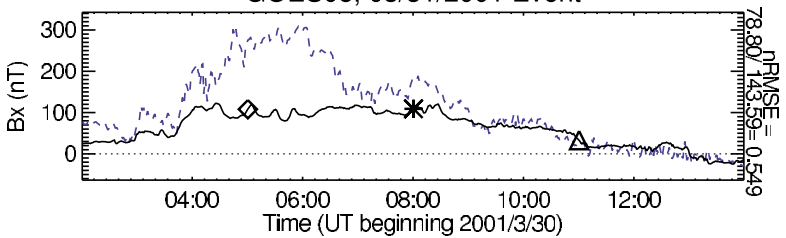

GOES08, 03/31/2001 Event

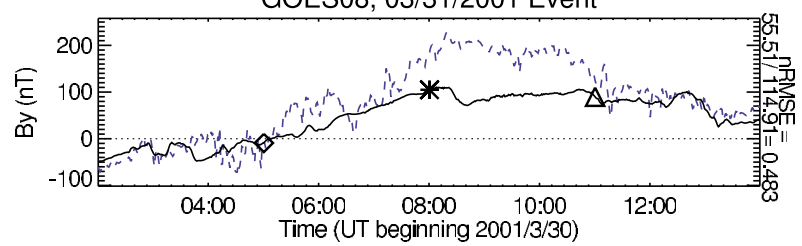

GOES08, 03/31/2001 Event
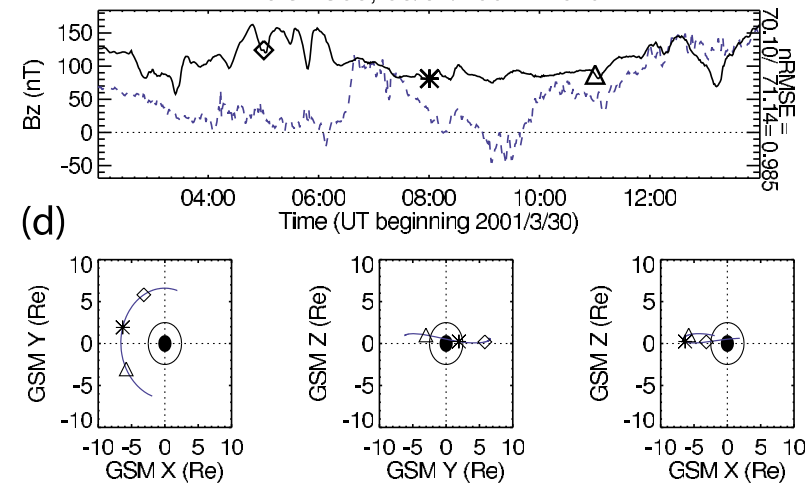

GOES10, 03/31/2001 Event
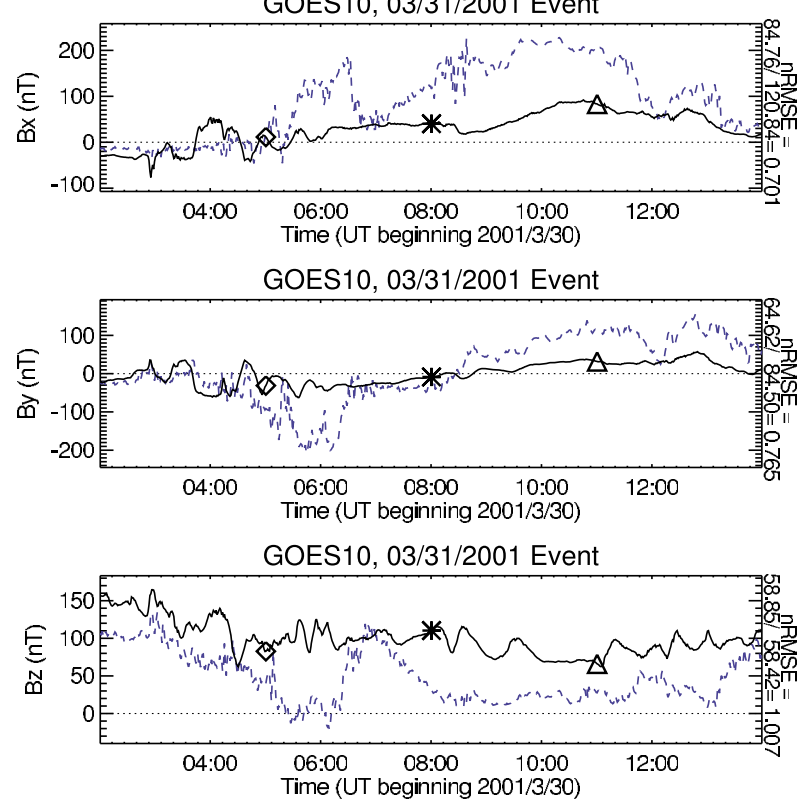

Figure 6. Data model comparisons of magnetic field from (a and c) GOES 08 and (b and d) GOES 10 with the MHD magnetic field calculated by BATS-R-US for the March 31, 2001 event. The data are in blue and the simulations are in black. Figures $6 \mathrm{a}$ and $6 \mathrm{~b}$ are from a multifluid MHD simulation with outflow and Figures $6 \mathrm{c}$ and $6 \mathrm{~d}$ are from a single-fluid MHD simulation without outflow. 
Table 1. Summary of the RMS Error Between the Data at the Various GOES Satellites and the Simulated Output at Those Locations ${ }^{\mathrm{a}}$

\begin{tabular}{|c|c|c|c|c|c|c|c|}
\hline \multirow[b]{2}{*}{ Event } & \multirow[b]{2}{*}{ Satellite } & \multicolumn{3}{|c|}{ RMS Error in $|B|$} & \multicolumn{3}{|c|}{ RMS Error in Elev $\Theta$} \\
\hline & & M-F MHD & M-S MHD & MHD & M-F MHD & M-S MHD & MHD \\
\hline \multirow{2}{*}{ May 4, 1998} & GOES 08 & 0.202 & 0.195 & 0.244 & 0.600 & 0.649 & 0.769 \\
\hline & GOES 09 & 0.217 & 0.214 & 0.299 & 0.680 & 0.728 & 1.094 \\
\hline \multirow[t]{2}{*}{ March 31, 2001} & GOES 08 & 0.227 & 0.180 & 0.312 & 0.691 & 0.631 & 0.983 \\
\hline & GOES 10 & 0.316 & 0.326 & 0.443 & 0.570 & 0.488 & 1.060 \\
\hline
\end{tabular}

${ }^{\mathrm{a}} \mathrm{M}-\mathrm{F}$ MHD and M-S MHD are multifluid MHD and multispecies MHD with ion outflow, respectively. The single fluid MHD control case does not include ion outflow.

comparison of our study. As Korth et al. [2004] report, the distribution function for $\mathrm{H}^{+}$and $\mathrm{O}^{+}$is quite complicated at this time, and strong counter streaming beams (with velocities exceeding $500 \mathrm{~km} / \mathrm{s}$ ) are seen in Cluster data after 8UT along field lines connected to the tail. While an MHD approach can still capture scalar values, such as density and pressure, it may be ill suited to studying the velocity where anisotropies in the distribution function have a more pronounced effect. The velocity differences may also be due to a problem in the coupling with the inner magnetosphere component (RCM model) which may need to be further improved. Further investigation including a wider range of simulated storms and velocity measurements are certainly needed to understand the limitations of the model.

[32] Figure 10 compares the $\mathrm{O}^{+}$and $\mathrm{H}^{+}$simulated pressure measurements to Cluster/CODIF data. The location and width of the simulated $\mathrm{O}^{+}$pressure peak is generally correct, as is the pressure after the peak. However, the magnitude of the simulated $\mathrm{O}^{+}$pressure peak is much too large. The reason that we apparently over predict the $\mathrm{O}^{+}$pressure peak has to do with the energy range of CIS/CODIF instrument on the Cluster satellite. The energy range for the instrument is from 40 to $40,000 \mathrm{eV}$ which excludes the significant contribution energetic ring current $\mathrm{O}^{+}$. When we recalculate the simulated $\mathrm{O}^{+}$pressure to only include particles in this energy range, we find an $\mathrm{O}^{+}$pressure that is a much closer match to the data. Our simulation also finds the peak in $\mathrm{H}^{+}$ pressure effectively. Unfortunately, when we restrict our calculation to the energy range of the instrument, the $\mathrm{H}^{+}$ pressure is much lower compared to the data. This indicates that while we adequately determine the $\mathrm{O}^{+}$pressure well, we predict an $\mathrm{H}^{+}$population that is hotter than the data would suggest.

[33] It is also interesting to examine the difference between the $\mathrm{H}^{+}$and $\mathrm{O}^{+}$velocities parallel and perpendicular to the magnetic field $\Delta u_{\|}=\left|\left(\mathbf{u}_{O^{+}}-\mathbf{u}_{H^{+}}\right) \cdot \mathbf{B} / B\right|$ and $\Delta u_{\perp}=$ $\left|\left(\mathbf{u}_{O^{+}}-\mathbf{u}_{H^{+}}\right) \cdot(I-\mathbf{B} / B)\right|$ These quantities are shown on the left-hand side of Figure 11; the right-hand side shows the magnetic field which matches quite well with the data. The velocity differences shown in Figure 11 exhibit some similarities to the Cluster/CODIF data, but could be improved. An interesting feature of the velocity differences is that deviation between the two species perpendicular to the magnetic field tracks the difference parallel to the field in the data, but not in the simulation. We speculate that the two-stream instability may be responsible for converting the large parallel velocity differences to the perpendicular direction in the data. The parallel velocity difference in the Cluster/CODIF measurements ranges from about $-130 \mathrm{~km} / \mathrm{s}$ to $150 \mathrm{~km} / \mathrm{s}$. In contrast, the magnitude of velocity difference in the perpendicular direction is alway less than $100 \mathrm{~km} / \mathrm{s}$ in the data. The similarities between the simulated and measured parallel velocity differences appears to justify the limitation placed on field-aligned velocity differences described in section 2.2.

\section{Conclusion and Discussion}

[34] We present in this study a new multifluid MHD version of the BATS-R-US magnetosphere model, including the equations solved, new algorithms and techniques, and some initial simulation results of geomagnetic storms. We compare the simulation results directly with the alternative techniques of multispecies and single-fluid MHD, and with a wide variety of data. The first results of our new multifluid model is found to perform well in initial tests modeling the May 4, 1998 and March 31, 2001 events.

[35] We compare our model results with large scale indices such as Dst and CPCP, and also with specific satellite measurements. Also included in the comparison are results, from Glocer et al. [2009], of alternative techniques of multispecies MHD (with ionospheric outflow) and traditional single-fluid MHD (without ionospheric outflow). The multifluid MHD is found to give similar results to the multispecies MHD in that both approaches give more strongly negative Dst, lower CPCP, and an improved comparison with GOES magnetic field compared with singlefluid MHD without outflow. Glocer et al. [2009] attribute these effects to ionospheric ions finding their way into the inner magnetosphere, strengthening the ring current and raising the plasma beta. The same mechanism is at work in this study. Additionally, both techniques similarly reproduce Cluster/CODIF composition data. We can therefore conclude that the inclusion of ionospheric outflow is more important than the additional physics we get from using the full multifluid MHD equations when studying the Dst index, the CPCP index, the magnetic field at geosynchronous orbit, or the composition in the inner magnetosphere. When studying composition or magnetic field further from the Earth, however, the multifluid and multispecies approaches begin to diverge. More work is needed to verify which approach more accurately represents the magnetosphere in these regions. We note that grid resolution in this region may play a role, since the outer magnetosphere is more coarsely resolved than the inner magnetosphere. However, initial tests found that increasing the resolution did not strongly affect the conclusions.

[36] Since all of the simulations considered here also include ring current effect through coupling with the inner magnetosphere model represented by RCM, it is reasonable to ask whether these effects are more important than the 


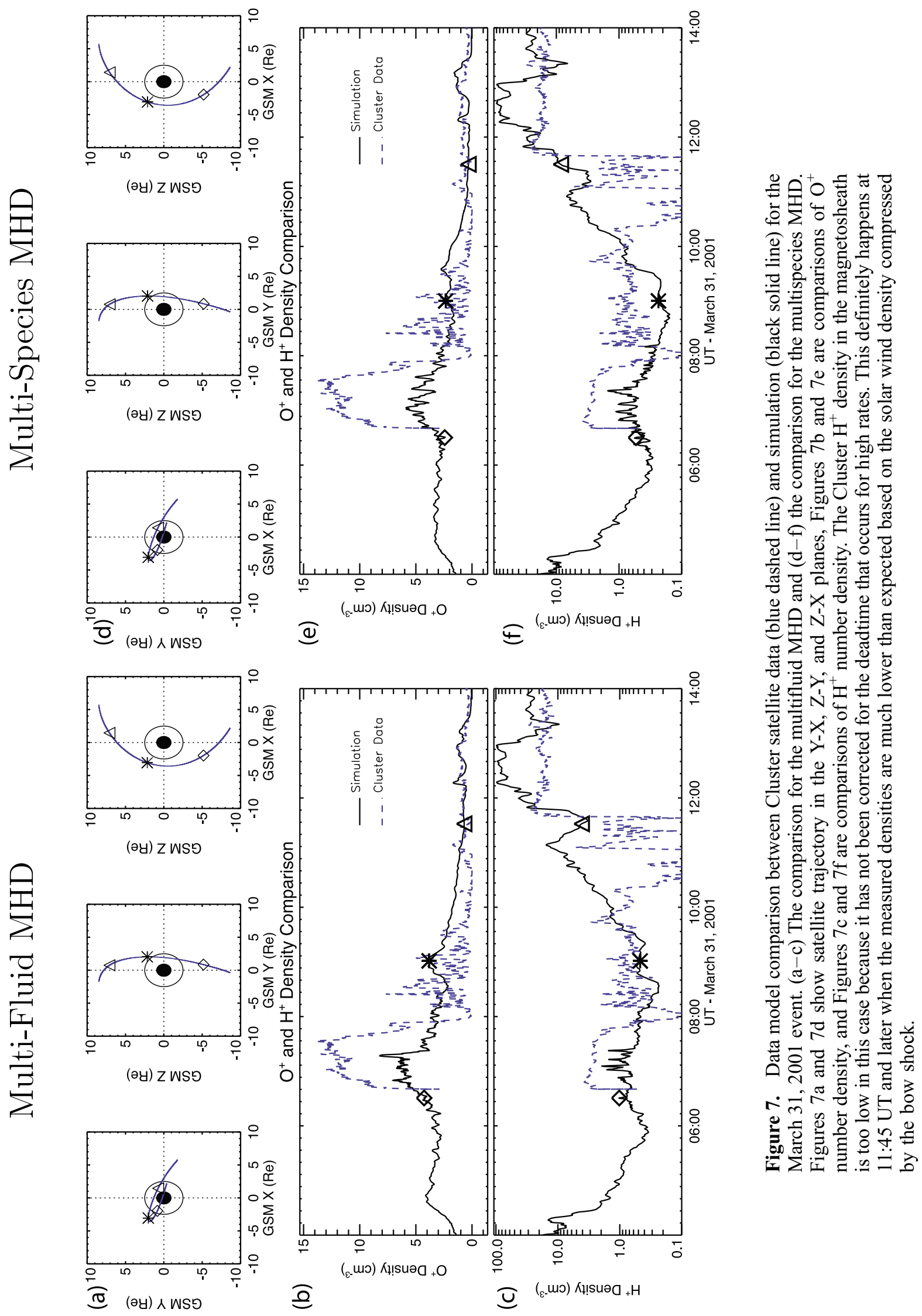




\section{3:00 UT}
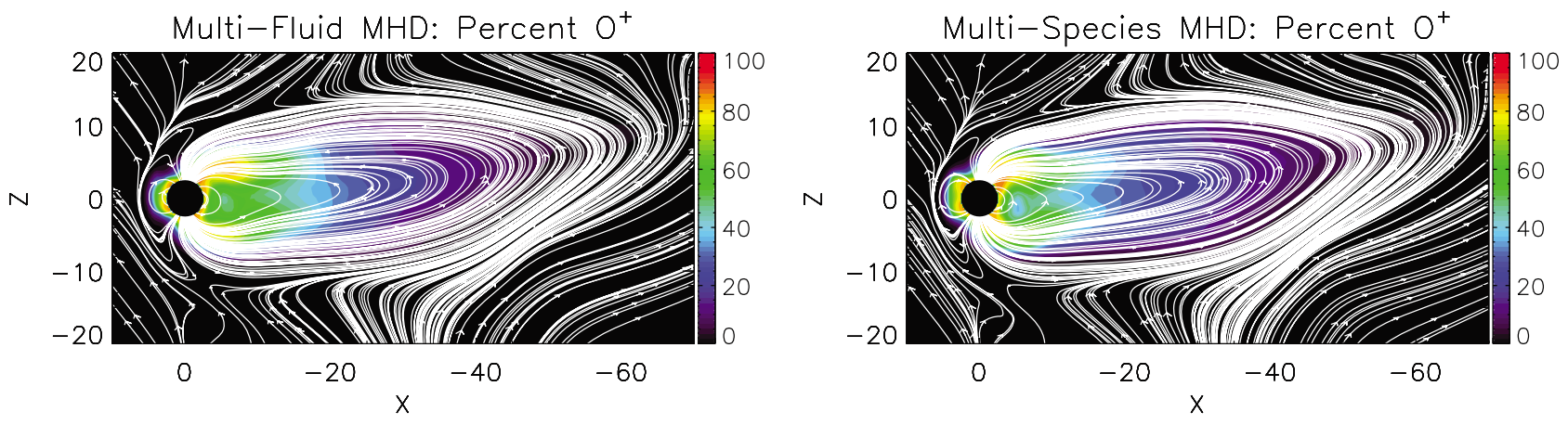

07:00 UT
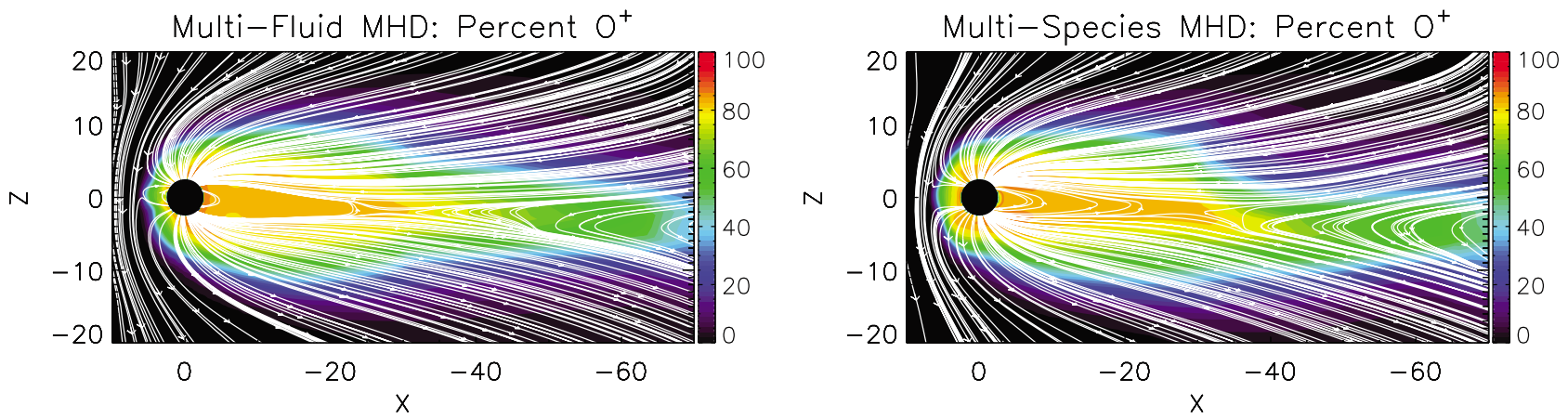

10:00 UT
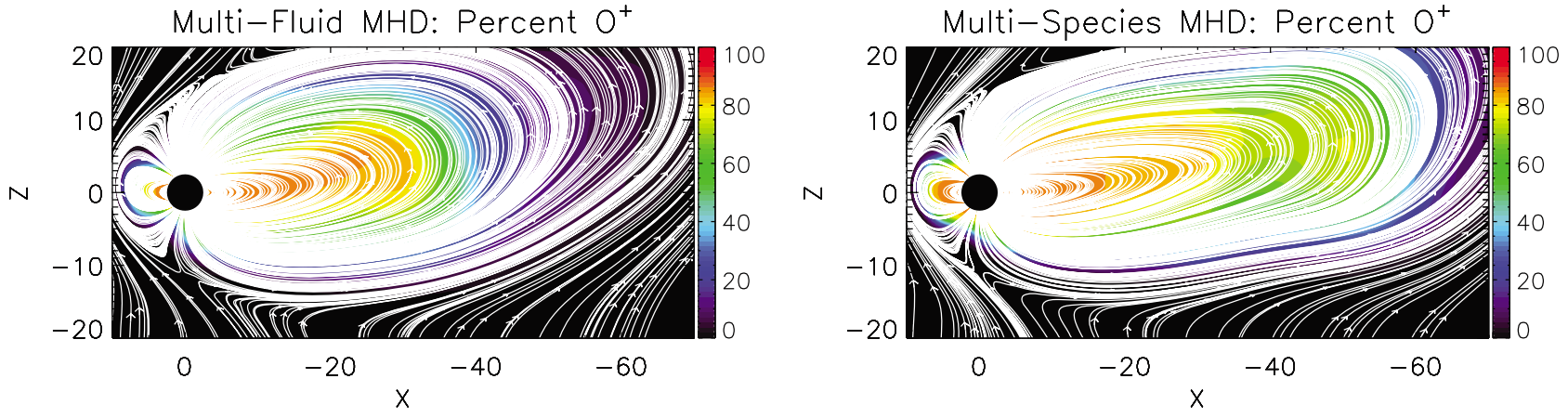

\section{3:00 UT}
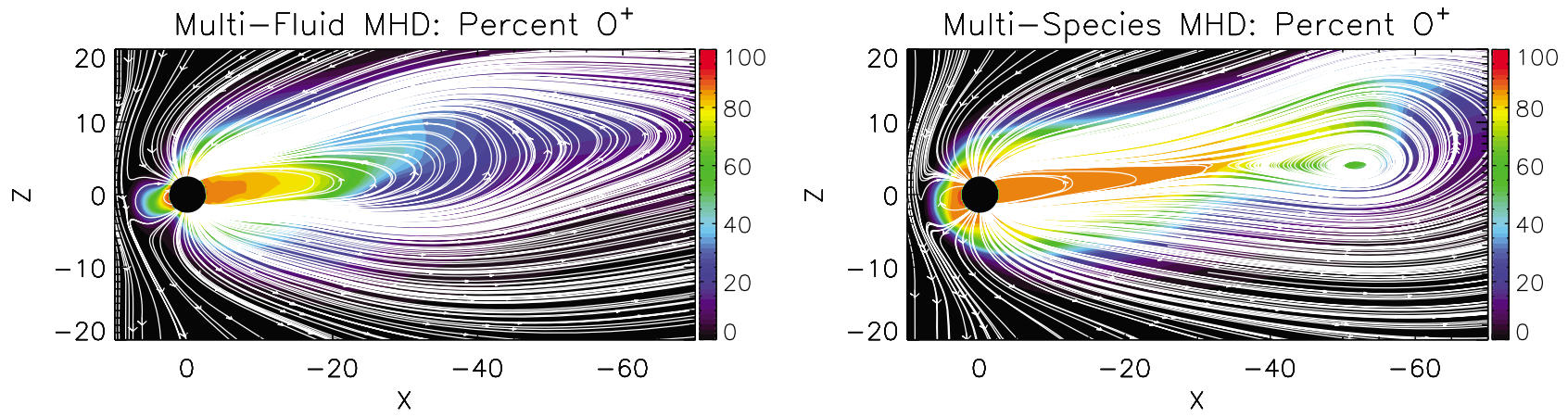

Figure 8. The percent of oxygen ions in the magnetosphere at 03:00, 07:00, 10:00, and 13:00 UT for the March 31, 2001 event. The $\mathrm{y}=0$ plane is shown. 


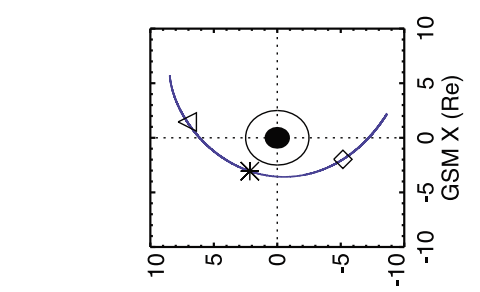

(әу) Z WSS

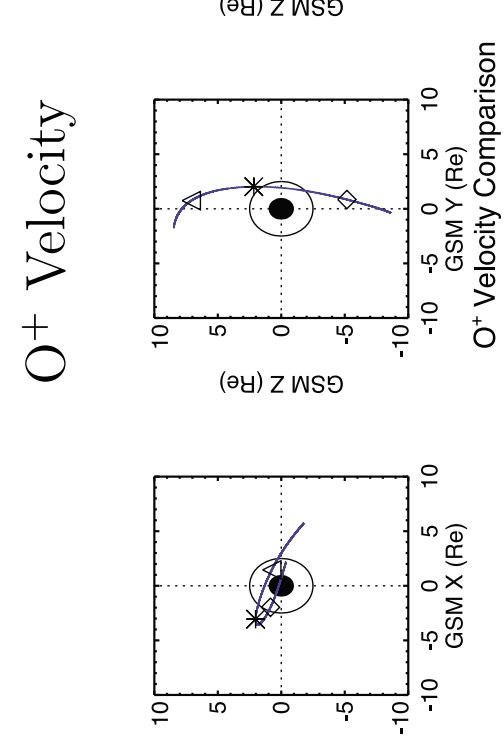

(1) (әу) ᄉ wsפ
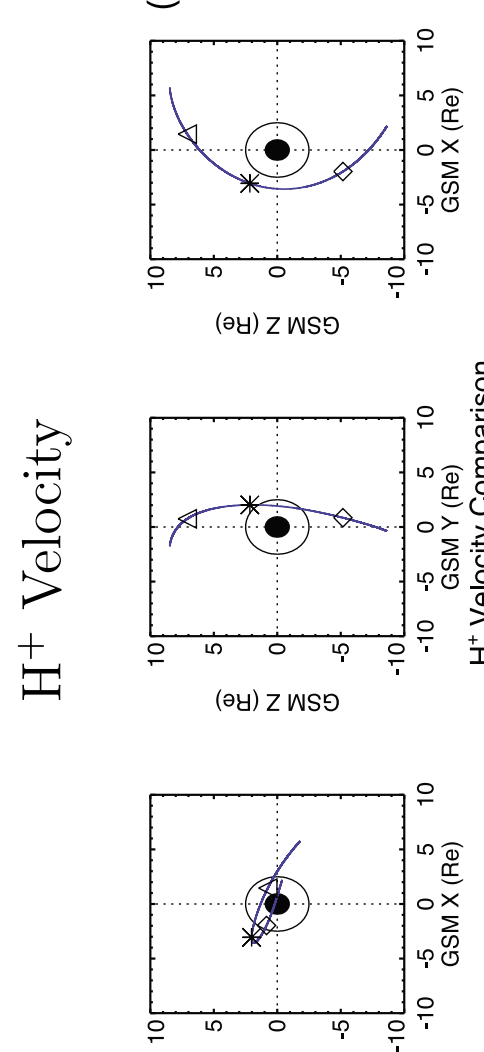

ฮี (әу) ᄉ WSפ

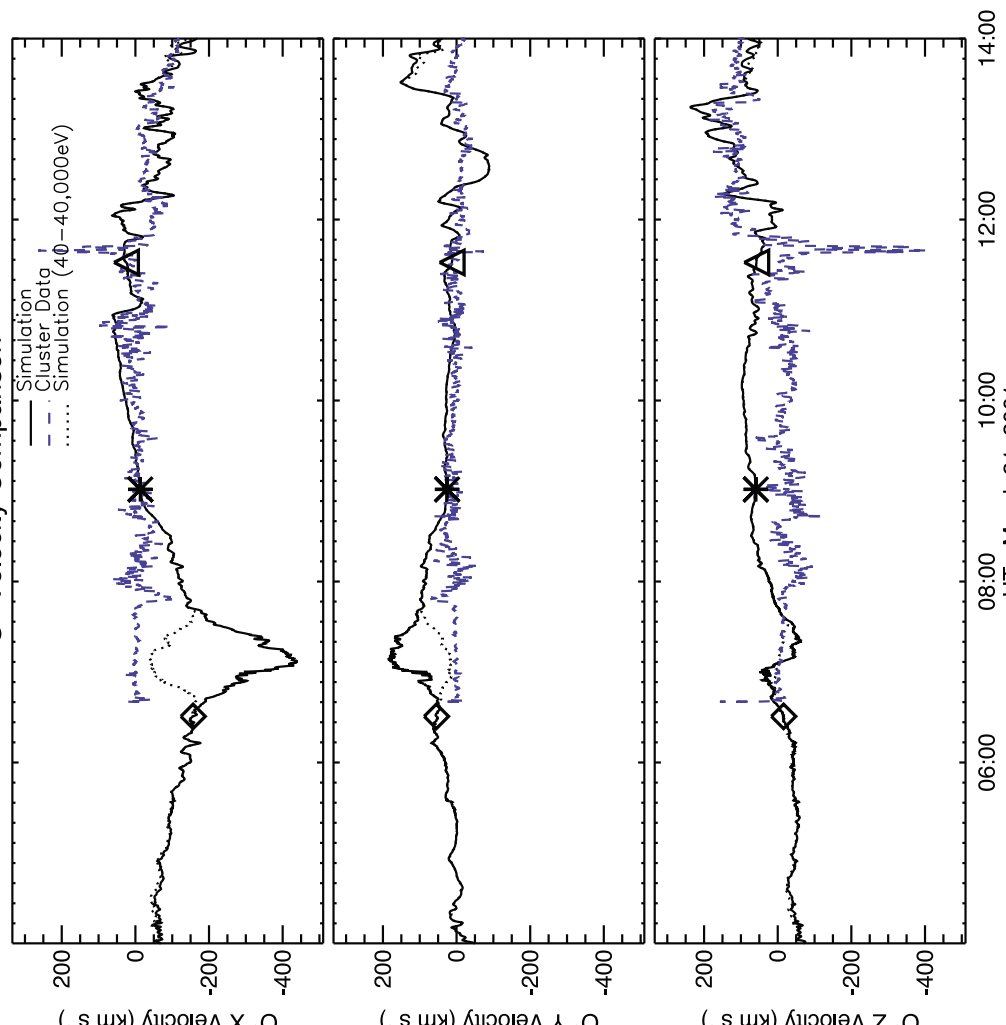

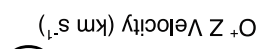

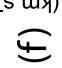

ㅁ

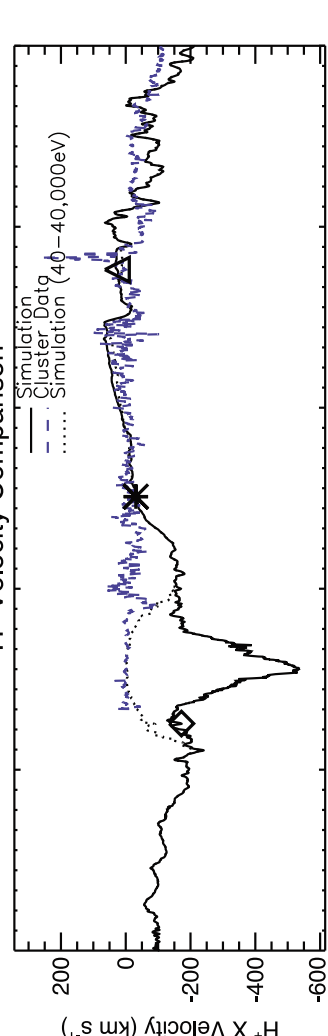

르

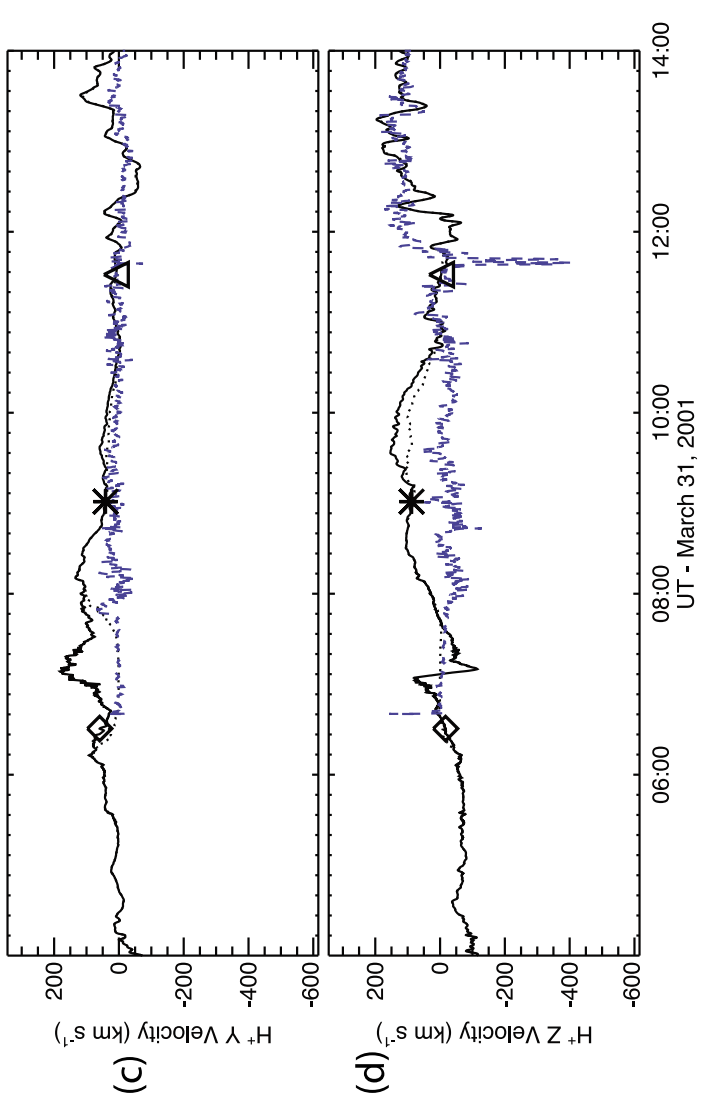

ธุำ

(ิ)

음 응

문

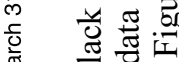

$\stackrel{\pi}{2}$ है

品

言离

言 导

का

究

可 :

윻

जै छे है

O

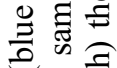

일

t马e

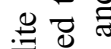

离

$\dot{0} \frac{0}{2}$

可

U.

ه

$\stackrel{0}{0}$ 远蓄

चี द्वे

क ज्ञ

늘 氖

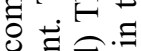

0 व $\partial^{2} \cdot$

क्षे

छे $\dot{0} \cdot \frac{.}{0}$

雨

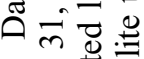

ब월 웅

닌 $\frac{4}{0}$

on 

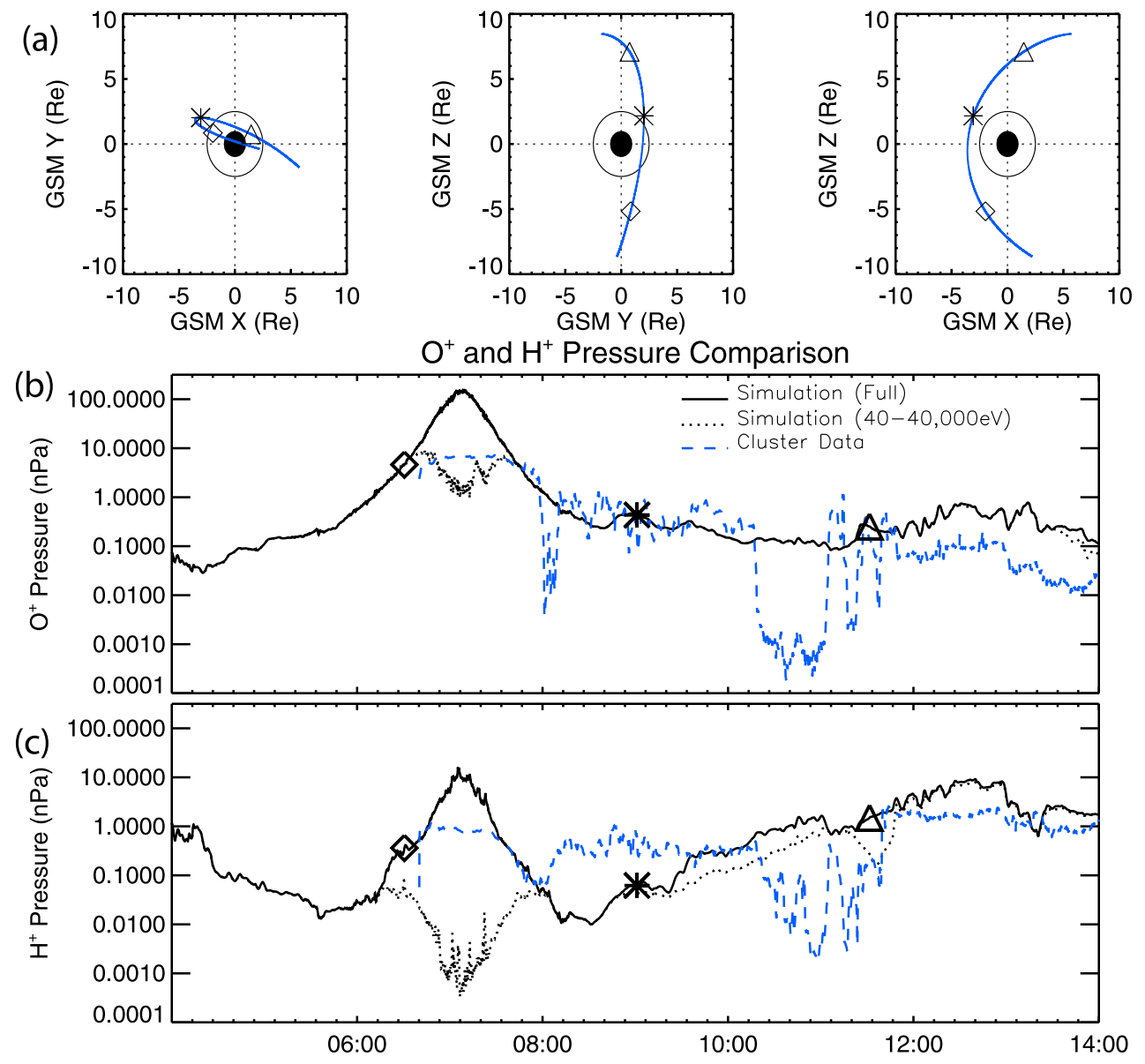

Figure 10. Data model comparison between Cluster satellite data (blue dashed line) and simulation (black solid line) for the March 31, 2001 event. The simulated pressure restricted to the same energy range as the satellite data is also shown (black dotted line). (a) The satellite trajectory in the Y-X, Z-Y, and $\mathrm{Z}$-X planes. The (c) $\mathrm{H}^{+}$and (b) $\mathrm{O}^{+}$pressure along the satellite trajectory.

inclusion of outflow. To address this concern, we repeated the simulations without the inclusion of ring current, and with ring current but without ionospheric outflow. We find that both ring current effects and ionospheric outflow are essential to modeling large storms. Neglecting either processes greatly reduces the quality of the results.

[37] Multifluid MHD opens the door to studies that are simply not available to the other techniques mentioned in this study. For instance, using our new model we can obtain individual ion velocities and pressures. This allows for the study of velocity differences between $\mathrm{H}^{+}$and $\mathrm{O}^{+}$. We assume in our model that streaming instabilities limit the flow difference parallel to the field and use an artificial friction to mimic that effect.

[38] The simulations in this study agree with other modeling and data studies that have been published. For instance, a study by Winglee et al. [2002], using a different multifluid MHD implementation, also found a strong reduction in the CPCP index. Observational studies of composition also are in accord with our results. Work by Lennartsson et al. [1981] examined composition data inside of $15 \mathrm{R}_{e}$ from the ISEE 1 satellite for 10 geomagnetic and found that $\mathrm{O}^{+}$can up as much as $80 \%$ of the entire ion population, a result consistent with our simulation.
[39] The model presented in this study differs from previous multifluid MHD models of the magnetosphere [Winglee, 2000; Winglee et al., 2002, 2005] in a number of ways. In the present study we attempt to calculate the ionospheric source directly instead of using centrifugal terms to fling $\mathrm{O}^{+}$into the magnetosphere. Moreover, we include coupling of the multifluid MHD model to an inner magnetosphere model (RCM) in order to capture a realistic storm time ring current. This last point is particularly important as we found that including ionospheric outflow does not have as strong an impact if ring current effects are not included. Finally, most BATS-R-US features such as Adaptive Mesh Refinement (AMR), ideal scaling when using multiple processors, and multiple total variation diminishing schemes are available in this new multifluid implementation.

[40] The initial results of our new model are extremely encouraging and demonstrate an ability to study problems not accessible by other methods. In future work we plan to further validate our model by carrying out more simulations that will allow us to compare $\mathrm{H}^{+}$and $\mathrm{O}^{+}$density, velocity, and pressure with satellite passes in a wider variety of locations in the magnetosphere. 

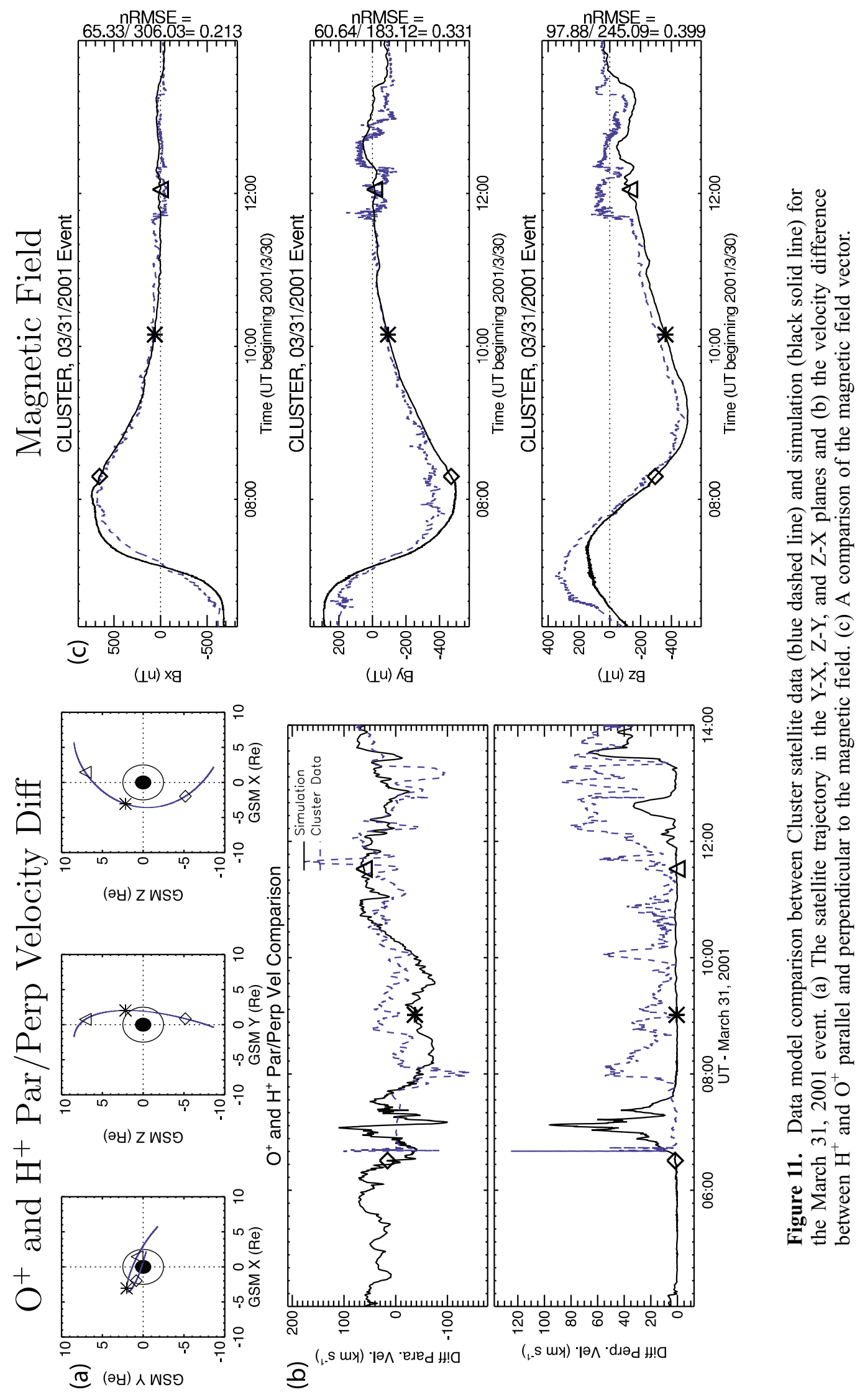
[41] Acknowledgments. This research was mainly funded by NASA grant NNX07AV80G. The Dst index is provided by World Data Center for Geomagnetism, Kyoto, and the Dst observatories (Kakioka, Honolulu, San Juan, Hermanus, and Alibag). We would also like to thank National Space Science Data Center for providing the ACE and GOES. ACE MAG instrument (N. F. Ness), SWEPAM instrument (D. McComas), and GOES Magnetometer (H. Singer). We would also like to acknowledge the CIS instrument team and ESA Cluster Active Archive for providing the Cluster data. Support for J. Zhang and L. Kistler was provided by NASA grant NNX07AP96G

[42] Zuyin Pu thanks the reviewers for their assistance in evaluating this manuscript.

\section{References}

Boris, J. P. (1970), A physically motivated solution of the Alfvén problem, Memo. Rep. 2167, Nav. Res. Lab., Washington, D. C.

Daglis, I. A., R. M. Thorne, W. Baumjohann, and S. Orsini (1999), The terrestrial ring current: Origin, formation, and decay, Rev. Geophys., 37, 407-438, doi:10.1029/1999RG900009.

De Zeeuw, D. L., T. I. Gombosi, C. P. T. Groth, K. G. Powell, and Q. F. Stout (2000), An adaptive MHD method for global space weather simulations, IEEE Trans. Plasma Sci., 28, 1956-1965.

De Zeeuw, D. L., S. Sazykin, R. Wolf, T. Gombosi, A. Ridley, and G. Tóth (2004), Coupling of a global MHD code and an inner magnetosphere model: Initial results, J. Geophys. Res., 109, A12219, doi:10.1029/ 2003JA010366.

Fu, S. Y., B. Wilken, Q. G. Zong, and Z. Y. Pu (2001), Ion composition variations in the inner magnetosphere: Individual and collective storm effects in 1991, J. Geophys. Res., 106, 29,683-29,704, doi:10.1029/ 2000JA900173.

Glocer, A., G. Toth, T. Gombosi, and D. Welling (2009), Modeling ionospheric outflows and their impact on the magnetosphere, initial results, J. Geophys. Res., 114, A05216, doi:10.1029/2009JA014053.

Gombosi, T. I., G. Tóth, D. L. De Zeeuw, K. C. Hansen, K. Kabin, and K. G. Powell (2001), Semi-relativistic magnetohydrodynamics and physicsbased convergence acceleration, J. Comput. Phys., 177, 176-205.

Huddleston, M. M., C. R. Chappell, D. C. Delcourt, T. E. Moore, B. L. Giles, and M. O. Chandler (2005), An examination of the process and magnitude of ionospheric plasma supply to the magnetosphere, J. Geophys. Res., 110, A12202, doi:10.1029/2004JA010401.

Kistler, L. M., et al. (2005), Contribution of nonadiabatic ions to the crosstail current in an $\mathrm{O}^{+}$dominated thin current sheet, J. Geophys. Res., 110, A06213, doi:10.1029/2004JA010653.

Korth, A., et al. (2004), Ion injections at auroral latitude during the March 31, 2001 magnetic storm observed by Cluster, Geophys. Res. Lett., 31, L20806, doi:10.1029/2004GL020356.

Lennartsson, W., R. D. Sharp, E. G. Shelley, R. G. Johnson, and H. Balsiger (1981), Ion composition and energy distribution during 10 magnetic storms, J. Geophys. Res., 86, 4628-4638, doi:10.1029/ JA086iA06p04628.

Lyon, J., J. Fedder, and C. Mobarry (2004), The Lyon-Fedder-Mobarry (LFM) global MHD magnetospheric simulation code, J. Atmos. Sol. Terr Phys., 66, 1333-1350.

Ma, Y. J., et al. (2007), 3D global multi-species Hall-MHD simulation of the Cassini T9 flyby, Geophys. Res. Lett., 34, L24S10, doi:10.1029/ 2007GL031627.

Moore, T. E., M.-C. Fok, D. C. Delcourt, S. P. Slinker, and J. A. Fedder (2007), Global aspects of solar wind ionosphere interactions, J. Atmos. Sol. Terr. Phys., 69, 265-278, doi:10.1016/j.jastp.2006.08.009

Nosé, M., R. W. McEntire, and S. P. Christon (2003), Change of the plasma sheet ion composition during magnetic storm development observed by the Geotail spacecraft, J. Geophys. Res., 108(A5), 1201, doi:10.1029/ 2002JA009660

Nosé, M., S. Taguchi, K. Hosokawa, S. P. Christon, R. W. McEntire, T. E. Moore, and M. R. Collier (2005), Overwhelming $\mathrm{O}^{+}$contribution to the plasma sheet energy density during the October 2003 superstorm: Geotail/EPIC and IMAGE/LENA observations, J. Geophys. Res., 110, A09S24, doi:10.1029/2004JA010930

Powell, K., P. Roe, T. Linde, T. Gombosi, and D. L. De Zeeuw (1999), A solution-adaptive upwind scheme for ideal magnetohydrodynamics, J. Comp. Phys., 154, 284-309.

Rème, H., et al. (2001), First multispacecraft ion measurements in and near the Earth's magnetosphere with the identical Cluster ion spectrometry (CIS) experiment, Ann. Geophys., 19, 1303-1354.

Ridley, A. J., and E. A. Kihn (2004), Polar cap index comparisons with amie cross polar cap potential, electric field, and polar cap area, Geophys. Res. Lett., 31, L07801, doi:10.1029/2003GL019113.

Ridley, A. J., T. Gombosi, and D. Dezeeuw (2004), Ionospheric control of the magnetosphere: Conductance, Ann. Geophys., 22, 567-584.

Seki, K., M. Hirahara, T. Terasawa, I. Shinohara, T. Mukai, Y. Saito, S. Machida, T. Yamamoto, and S. Kokubun (1996), Coexistence of Earth-origin $\mathrm{O}^{+}$and solar wind-origin $\mathrm{H}^{+} / \mathrm{He}^{++}$in the distant magnetotail, Geophys. Res. Lett., 23, 985-988, doi:10.1029/96GL00768.

Sharp, R. D., W. Lennartsson, and R. J. Strangeway (1985), The ionospheric contribution to the plasma environment in near-earth space, Radio Sci., 20, 456-462.

Shelley, E. G., R. G. Johnson, and R. D. Sharp (1972), Satellite observations of energetic heavy ions during a geomagnetic storm, J. Geophys. Res., 77, 6104-6110, doi:10.1029/JA077i031p06104.

Stout, Q. F., D. L. De Zeeuw, T. I. Gombosi, C. P. T. Groth, H. G. Marshall, and K. G. Powell (1997), Adaptive blocks: A high-performance data structure, paper presented at 1997 Conference on Supercomputing, Assoc. of Comput. Mach. and Inst. of Electr. and Electr. Eng., San Jose, Calif.

Tóth, G., et al. (2005), Space weather modeling framework: A new tool for the space science community, J. Geophys. Res., 110, A12226, doi:10.1029/ 2005JA011126.

Tóth, G., D. L. De Zeeuw, T. I. Gombosi, and K. G. Powell (2006), A parallel explicit/implicit time stepping scheme on block-adaptive grids, J. Comput. Phys., 217, 722-758, doi:10.1016/j.jcp.2006.01.029.

Tóth, G., Y. J. Ma, and T. I. Gombosi (2008), Hall magnetohydrodynamics on block adaptive grids, J. Comput. Phys., 227, 6967-6984, doi:10.1016/j.jcp.2008.04.010.

Wang, H., A. J. Ridley, and H. Luhr (2008), Validation of the Space Weather Modeling Framework using observations from CHAMP and DMSP, Space Weather, 6, S03001, doi:10.1029/2007SW000355.

Winglee, R. M. (2000), Mapping of ionospheric outflows into the magnetosphere for varying IMF conditions, J. Atmos. Sol. Terr. Phys., 62, $527-540$

Winglee, R. M., D. Chua, M. Brittnacher, G. K. Parks, and G. Lu (2002), Global impact of ionospheric outflows on the dynamics of the magnetosphere and cross-polar cap potential, J. Geophys. Res., 107(A9), 1237, doi:10.1029/2001JA000214.

Winglee, R. M., W. Lewis, and G. Lu (2005), Mapping of the heavy ion outflows as seen by IMAGE and multifluid global modeling for the 17 April 2002 storm, J. Geophys. Res., 110, A12S24, doi:10.1029 2004JA010909.

A. Glocer, NASA GSFC, Mail Code 673, Greenbelt, MD 20771, USA (alex.glocer-1@nasa.gov)

T. Gombosi and G. Tóth, Department of Atmospheric Oceanic and Space Science, University of Michigan, 2455 Hayward St., Ann Arbor, MI 48109-2143, USA.

L. M. Kistler and J.-C. Zhang, Space Science Center, University of New Hampshire, 8 College Rd., Durham, NH 03824, USA.

Y. Ma, Institute of Geophysics and Planetary Physics, UCLA, 3845 Slichter Hall, Charles E. Young Dr., E, Los Angeles, CA 90065-1567, USA. 